\title{
Costs and benefits of transgenerational acquired resistance in Arabidopsis.
}

\author{
Ana Lopez Sanchez ${ }^{1}$, David Pascual Pardo ${ }^{1}$, Leonardo Furci ${ }^{1}$, Michael Roberts ${ }^{2}$, and \\ Jurriaan Ton ${ }^{1}$ \\ ${ }^{1}$ University of Sheffield \\ ${ }^{2}$ Lancaster University
}

October 2, 2020

\begin{abstract}
Recent evidence suggests that stressed plants employ epigenetic mechanisms to transmit acquired resistance to their progeny. However, little is known about the evolutionary and ecological significance of this transgenerational acquired resistance (TAR). In this study, we have used a full factorial design to study the specificity, costs and stability of TAR following exposure of Arabidopsis thaliana to increasing stress intensities by a biotrophic pathogen, a necrotrophic pathogen, and soil salinity. All stresses incrementally reduced parental growth, while salt stress additionally impacted reproductive success. Biotrophic and necrotrophic pathogens, but not salt, increased resistance of progeny against the stress experienced by their parents (i.e., in matched environments). In mis-matched environments, however, pathogen-elicited TAR was associated with costs from increased susceptibility to other stresses. Furthermore, the stability of pathogen-elicited TAR over one stress-free generation and its associated costs were proportional to parental disease severity, suggesting that plants use stress intensity as an environmental proxy to adjust TAR investment. We conclude that pathogen-elicited TAR is an adaptive and deterministic parental effect that is associated with ecological costs. Accordingly, our study provides evolutionary and ecological context to the epigenetic TAR response.
\end{abstract}

Key words: Adaptive parental effects; Arabidopsis; Phenotypic plasticity; Plant stress; Transgenerational acquired resistance.

\section{Introduction}

Phenotypic plasticity allows organisms to modify their biochemical, physiological or morphological traits to survive in changing environments (Schlichting, 1986). (Lämke \& Bäurle, 2017; Bošković \& Rando, 2018). These epigenetic responses have the potential to provide adaptive benefits to progeny, thereby enhancing evolutionary fitness of the parents. When facing environmental changes, organisms can adopt various transgenerational strategies to optimise their fitness. When environments change frequently and are unpredictable, parents may adopt a bet-hedging strategy to increase the variability within their progeny (Crean \& Marshall, 2009). By contrast, when environments undergo directional and stable changes, which present 
a more predictable cue about future environmental conditions, parents could enhance reproductive fitness by transmitting specific adaptive traits to their progeny (Marshall \& Uller, 2007; Proulx \& Teotonio, 2017).

Transgenerational responses to stress have been reported in both plants and animals; ranging from maladaptive pathological effects of environmental pollutants, to adaptive immunological traits that increase resistance against pests and diseases (Holeski et al., 2012; Luna et al., 2012; Rasmann et al., 2012; Perez \& Lehner, 2019; Tetreau et al., 2019). In plants, transgenerational induced resistance to disease is often referred to as 'transgenerational acquired resistance' (TAR), which is typically based on a sensitisation, or 'priming', of the immune system, mediating a faster and/or stronger immune response (Wilkinson et al., 2019). We have previously demonstrated that bacterial speck disease, caused by the hemi-biotrophic pathogen Pseudomonas syringae pv. tomato (Pst), elicits TAR that can be maintained over two stress-free generations in the self-fertilising annual plant Arabidopsis (Arabidopsis thaliana) (Luna et al., 2012; Stassen et al., 2018). Although the exact epigenetic mechanisms underpinning TAR are still under investigation, the induction and/or transmission of the resistance requires DNA demethylation at transposable elements, and is associated with genome-wide changes in DNA methylation (Luna \& Ton, 2012; Lopez Sanchez et al., 2016; Stassen et al., 2018; Furci et al., 2019). These results are supported by numerous other studies reporting transgenerational changes in DNA methylation in response to environmental stress (Lämke \& Bäurle, 2017; Wilkinson et al., 2019).

Despite the fact that epigenetic modifications are attracting much attention as a mechanism for transgenerational phenotypic plasticity, there is still controversy over whether such responses are adaptive (Herman \& Sultan, 2011; Uller et al., 2013; Burggren, 2015; Crisp et al., 2016). Transgenerational phenotypic plasticity to abiotic conditions, such as light and water availability, have been shown to provide improved fitness when the environments of parents and progeny are matched (Galloway \& Etterson, 2007; Herman et al., 2012). However, when parent and progeny environments are mismatched, transgenerational effects can be maladaptive, which may explain why many epigenetic modifications are erased during sexual reproduction (Iwasaki \& Paszkowski, 2014; Crisp et al., 2016; Gehring, 2019). For TAR to be genuinely adaptive, theory provides three key predictions. First, TAR should be elicited by specific stresses that the parents can recover from and that generate corresponding specific phenotypic changes in their progeny. Secondly, since TAR is an inducible response, there should be associated costs that may only become apparent in mismatched environments. Finally, parents should have the ability to distinguish strong, reliable cues with high predictive value about future environments. There is limited evidence to support some of these predictions. For instance, while progeny from $P$. syringae-infected Arabidopsis exhibit TAR against another biotrophic pathogen, Hyaloperonospora arabidopsidis ( $\mathrm{Hpa}$ ), this same progeny showed enhanced susceptibility to the necrotrophic fungus Alternaria brassicicola (Luna et al., 2012). Similarly, progeny from spider mite-infested Arabidopsis were primed for defence against both spider mites and aphids but suffered increased susceptibility to $P$. syringae (Singh et al., 2017). However, none of these studies employed reciprocal designs to systematically test predictions relating to specificity and costs within a single experimental framework. Moreover, it remains unknown whether plants can perceive stress intensity as an environmental proxy to estimate the likelihood that the same stress is still present in the progeny environment and adjust the strength and/or durability of TAR accordingly.

Here, we have addressed the above hypotheses by characterising TAR responses of Arabidopsis to different types and intensities of biotic and abiotic stress. Using a full factorial reciprocal experimental design, we have examined the specificity of TAR by quantifying the impacts of three parental stresses in both matched and mismatched progeny environments. We show that TAR elicited by biotrophic and necrotrophic pathogens is not associated with reductions in reproductive fitness and provides resistance benefits to progeny in matched environments. However, these specific TAR responses were associated with ecological costs that become apparent in mismatched environments from enhanced susceptibility to other stresses. By contrast, abiotic stress by soil salinity failed to elicit TAR against salt but elicited non-specific resistance in mismatched environments against biotrophic and necrotrophic pathogens, which was offset by major reproductive costs from dramatically reduced seed production and viability. Finally, we demonstrate that the transgenerational stability and costs of pathogen-elicited TAR are proportional to the disease intensity experienced by the 
parents and discuss these results in context of current evolutionary theory about transgenerational phenotypic plasticity.

\section{Results}

\section{Dose-dependent effects of biotic and abiotic stresses on plant fitness parameters.}

To test our central hypotheses, we produced populations of Arabidopsis progeny that in the parental generation had been exposed to three different stresses: the (hemi)biotrohic pathogen Pseudomonas syringae pv. tomato DC3000 (Pst), the necrotrophic pathogen Plectosphaerella cucumerina (Pc), and salt stress (Fig. 1). All parents came from a single common ancestor to minimise (epi)genetic variation. For each stress type, we applied four severity levels (mock plus three increasing levels of the stress) and estimated their effects on different fitness parameters (Fig. 2).

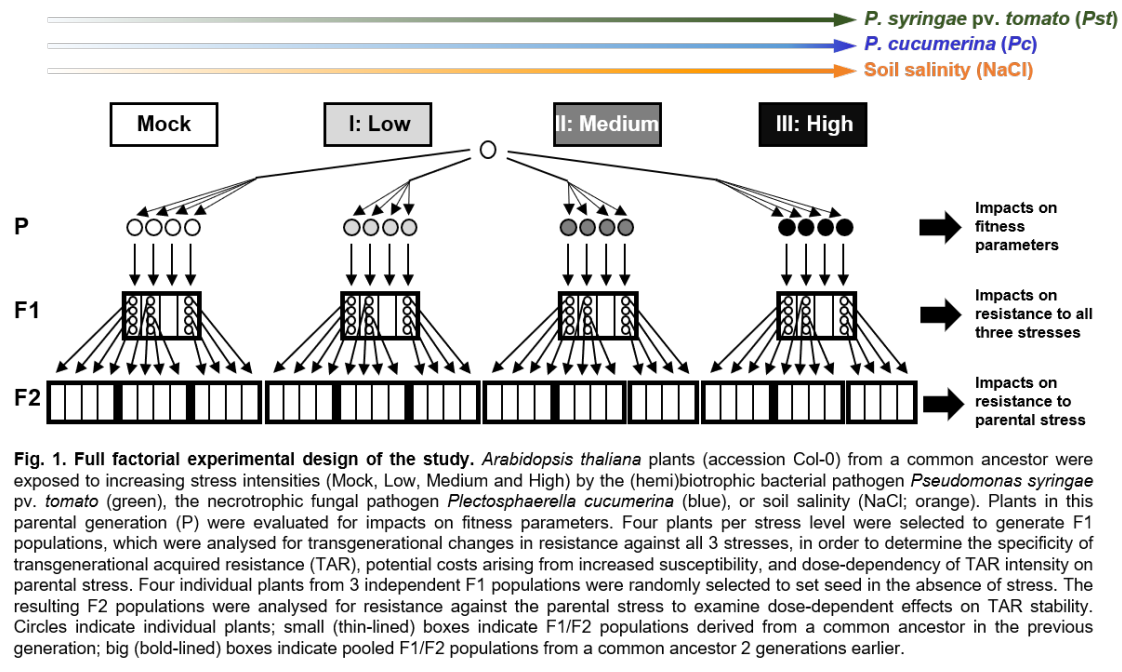

Figure 1: This is a caption

All stresses induced a dose-dependent decline in relative growth rate (RGR), confirming that the plants perceived and responded to the stresses in a dose-dependent manner (Fig. 2a). By contrast, seed production showed a different response to the stresses. The lowest levels of disease by Pst and Pc stimulated seed production, whereas the highest stress levels by these diseases had no statistically significant effect on seed production (Fig. 2b). This suggests that Arabidopsis can adapt to both diseases by compensating the reduced growth during pathogen exposure with increased seed production at the end of its life cycle. Conversely, increasing levels of soil salinity caused a dose-dependent reduction in seed production (Fig. 2b), indicating that Arabidopsis does not recover as efficiently from this stress as it does from disease by Pst or Pc. Similar patterns were observed for seed viability, where $P s t$ and $P c$ failed to have an effect (Fig. 2c and Fig. S1a,b), whereas soil salinity caused a dramatic dose-dependent decline in seed viability (Fig. 2c and Fig. S1c), which was absent in F2 seeds after one stress-free F1 generation (Fig. S1d). 
(a)

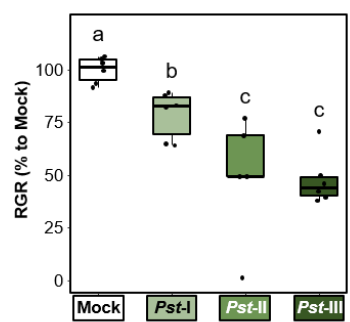

(b)

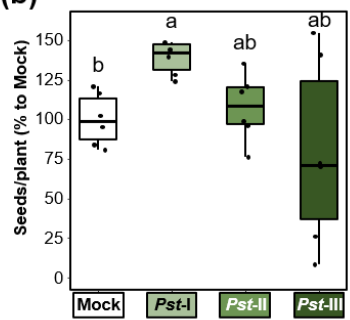

(c)

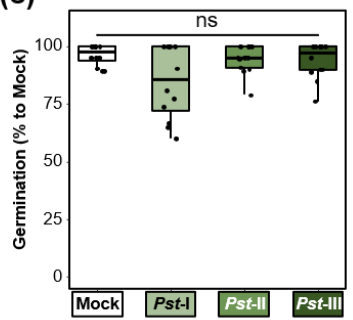

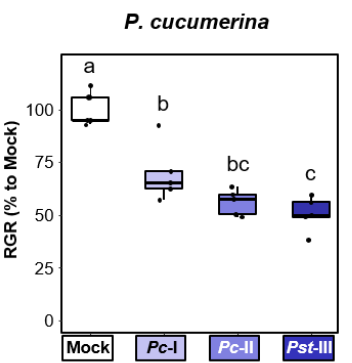
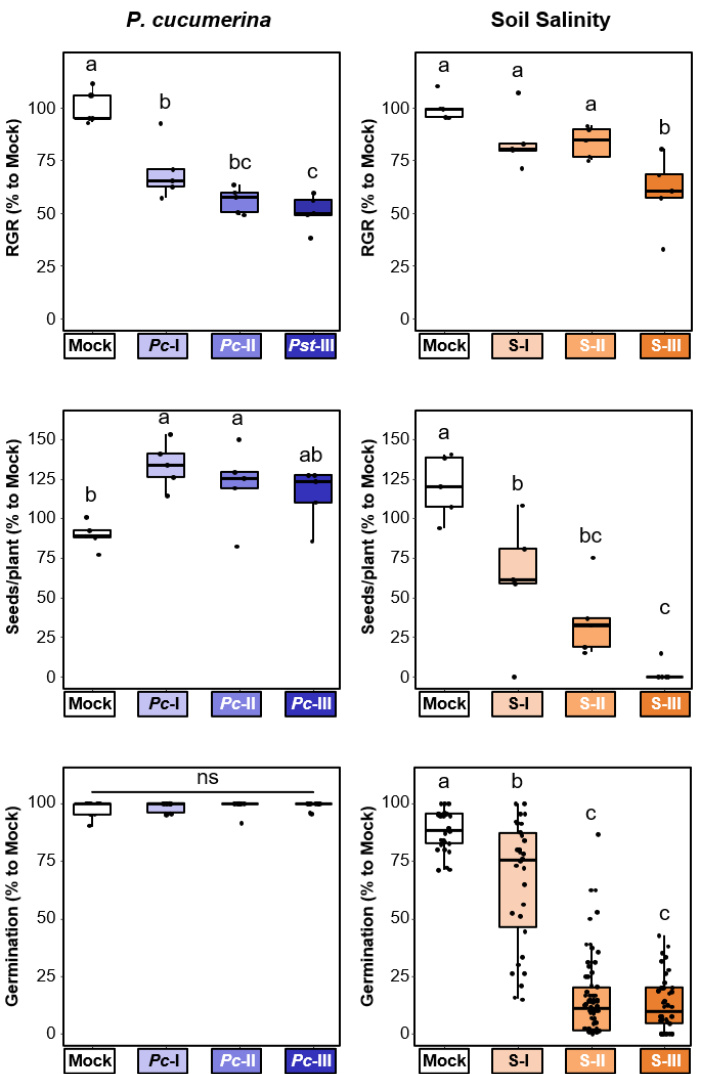

Fig. 2. Differential impacts of three (a)biotic stresses on parental fitness parameters. Plants in the parental generation (4.5weeks-old) were exposed to varying stress intensities by $P$. syringae pv tomato (Mock, Pst-I, Pst-II, Pst-III), P. cucumerina (Mock, Pc-I, Pc-II, Pc-III) or soil salinity (Mock, S-I, S-II, S-III) over a 3-week period before transferring to long-day conditions to trigge flowering and set seed. Boxplots show the interquartile range (IQR; box) $\pm 1.5 \times I Q R$ (whiskers), including median (horizonal line) and replication units (single dots). (a) Impacts on relative growth rate (RGR) during the period of stress exposure. Data represent RGR values of single plants $(n=5-6)$ normalised to the average RGR of Mock-treated plants $(100 \%)$. Different letters indicate statistically significant differences (ANOVA + Tukey post-hoc test, $a=0.05$ ). (b) Impacts on seed production. Data represent seed numbers per plant $(n=5-6)$ normalised to average value of Mock-treated plants $(100 \%)$. Different letters indicate statistically significant differences (Pst: Welch ANOVA + Games-Howell posthoc test, $\alpha=0.05 ; P c$ and salt: ANOVA + Tukey post-hoc test, $\alpha=0.05)$. (c) Impacts on seed viability. Seed viability was determined 5 days after planting of surface-sterilised and stratified seeds a 0.05$)$ (c) Murashige and Skoog (MS) agar plates. Das determited seeds onto $0.2 \times$ Murashige and Skoog (MS) agar plates. Data represent mean germination percentages per plate $(25 \mathrm{seeds} / \mathrm{plate})$ of ANOVA + Games-Howell post-hoc test; $a=0.05$ ). Viability data for seed batches from individual plants are presented in Fig. S1a-c.

Figure 2: This is a caption

\section{Parental stress leads to beneficial or neutral impacts on resistance of progeny in matched environments.}

Next, we investigated TAR in F1 progeny against the same stress to which the parents had been exposed (matched environments). Parents exposed to disease by biotrophic Pst produced F1 progeny that were more resistant to both Pst (Fig. 3a and Fig. S2a), and the biotrophic Oomycete Hyaloperonospora arabidopsidis (Hpa; Fig. 3b and Fig. S2b). These findings support our previous results (Luna et al., 2012) and demonstrate that Pst-elicited TAR is not specific at the level of pathogen species, but that it protects against taxonomically unrelated pathogens with similar biotrophic lifestyles. 
(a) Parents Pst - F1 Pst

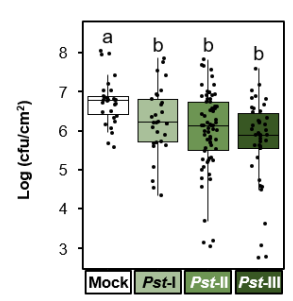

(b) Parents Pst - F1 Hpa

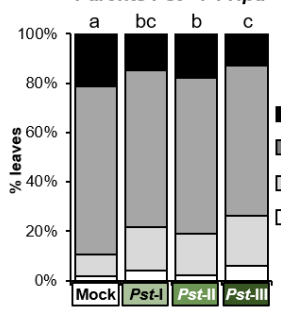

(c)

Parents Pst - F2 Hpa

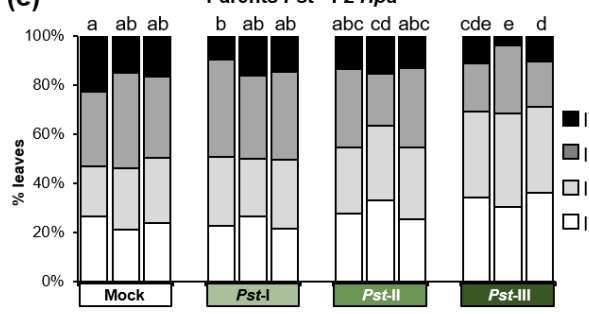

Fig. 3. Intensity and transgenerational stability of Pseudomonas symgae pv. tomat environments. Parental plants had been exposed to different disease severities by the biotrophic bacterium Pseudomonas syringae pv. tomato (Pst, Mock, Pst-I, Pst-II, Pst-III). F1 and F2 plants were analysed for resistance against the same pathogen (Pst) and/or the biotrophic Oomycete Hyaloperonospora arabidopsidis ( $\mathrm{Hpa}$ ). (a) TAR against Pst in F1 progeny at 3 days post infection (dpi). Boxplots show the interquartile range (IQR; box) $\pm 1.5 x \mathrm{IQR}$ (whiskers), including median (horizonal line) and replication units (dots). Data represent ${ }^{10} \mathrm{Log}-$ transformed bacterial titres ( $\log \mathrm{cfu}_{\mathrm{cm}}^{-2}$ ) in leaves of single plants within F1 populations from similarly treated parents $(n=42)$. Different letters indicate statistically significant differences (Welch ANOVA + Games-Howell test, $\alpha=0.05$ ). Data for individal F1 populations are shown in Fig. S2a. (b) TAR against Hpa in $\mathrm{Fr}$ progeny. Hpa colonisation was quantified at 6 dpi by assigning trypan-blue stained leaves to 4 Hpa resistance classes (I. healty, I. hyphal colonisation only; III hyphal conization with conidiospores; IV hyphal collonisation with distributions within $F 1$ popuations from sim distribulions win statistically significant differences (Painvise Fisher's exact tests - Bonferroni FDR $\alpha=0.05$ ). Data for individual F1 populations are shown in Fig S2b. (c) TAR against Hpa in F2 progeny at 6 dpi after one stress-free $\mathrm{F} 1$ generation. Stacked bars show leaf frequency distributions across $H p a$ resistance classes within $F 2$ populations that share a common parental ancestor $(n=300$ 350). Different letters indicate statistically significant differences (Pairwise Fisher's exact tests + Bonferroni FDR; $\alpha=0.05$ ). Data for individual F2 populations are shown in Fig. S2C.

Figure 3: This is a caption

To determine whether Arabidopsis develops TAR in response to necrotrophic pathogens, we tested F1 progeny from parents exposed to increasing disease by the necrotrophic fungus $P c$ for resistance against the same pathogen. Compared to progeny from mock-inoculated parents, all but the lowest severity of parental disease resulted in a statistically significant suppression of $P c$ lesion development in F1 progeny (Fig. 4a and Fig. S3a). 


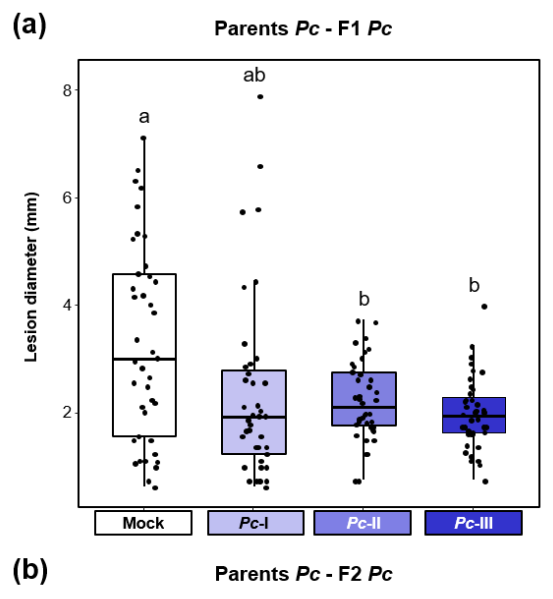

Fig. 4. Intensity and transgenerational stability of Plectosphaerella cucumerina-elicited TAR in matched environments. Parental plants had been exposed to different disease severities by necrotrophic Plectosphaerella cucumerina (Pc; Mock, Pc-I, Pc-II, Pc-III). F1 and F2 plants were analysed for resistance against the same pathogen. Lesion diameters were determined in 4 leaves/plant at 15 days post infection (dpi) and the average lesion diameter per plant was used as statistical unit of replication. Boxplots show the interquartile range (IQR; box) \pm $1.5 \times I Q R$ (whiskers), including median (horizonal line) and replication units (dots). (a) TAR against $P C$ in F1 progeny. Data represent lesion diameters $(\mathrm{mm})$ of plants within $\mathrm{F} 1$ populations represent lesion diameters $(\mathrm{mm})$ of plants within F1 populations
from similarly treated parents $(\mathrm{n}=40)$. Different letters indicate from similarly treated parents $(\mathrm{n}=40)$. Different letters indicate statistically significant differences (Welch ANOVA + Games-Howell s3a. (b) TAR against Pc in F2 progeny after a stress-free F1 generation. Data represent lesion diameters of plants within F2 gepultions that share a common parental ancestor ( $n=20)$. Different letters indicate statistically significant difference (ANOVA + Tukey posthoc test: $\alpha=0.05$ ). Data for individual F2 populations are shown in Fig. S3b.

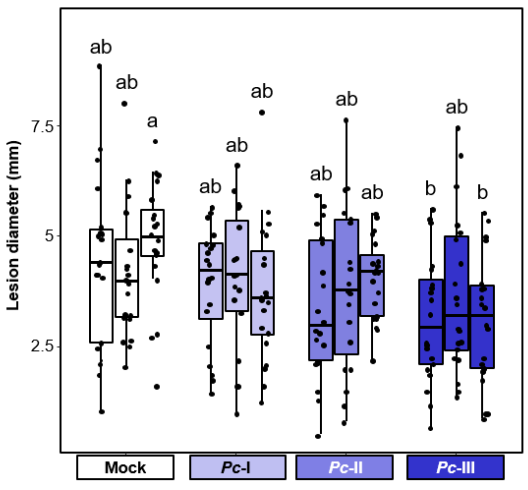

Figure 4: This is a caption

Finally, we investigated the transgenerational effects of soil salinity in matched environments. To this end, $\mathrm{F} 1$ progeny from parents exposed to increasing $\mathrm{NaCl}$ concentrations in the soil were analysed for root growth inhibition on agar medium supplemented with $50 \mathrm{mM}$ and $100 \mathrm{mM} \mathrm{NaCl}$, which is a common method to quantify salt tolerance in Arabidopsis (Verslues et al., 2006; Claeys et al., 2014). F1 populations from differently treated parents showed small but statistically significant differences in root growth on agar medium containing $0 \mathrm{mM}$ and $50 \mathrm{mM} \mathrm{NaCl}$ (Fig. S4a), which were absent in the F2 generation (Fig. S4b). However, these differences in root growth appeared non-adaptive, since the degree of NaCl-induced root growth inhibition compared to roots on control plates $(0 \mathrm{mM} \mathrm{NaCl})$ was similar between populations from all parental treatments (Fig. 5a,b and Fig. S4c,d). Thus, under our conditions, progeny from salt-stressed plants did not express TAR in matched environments. 

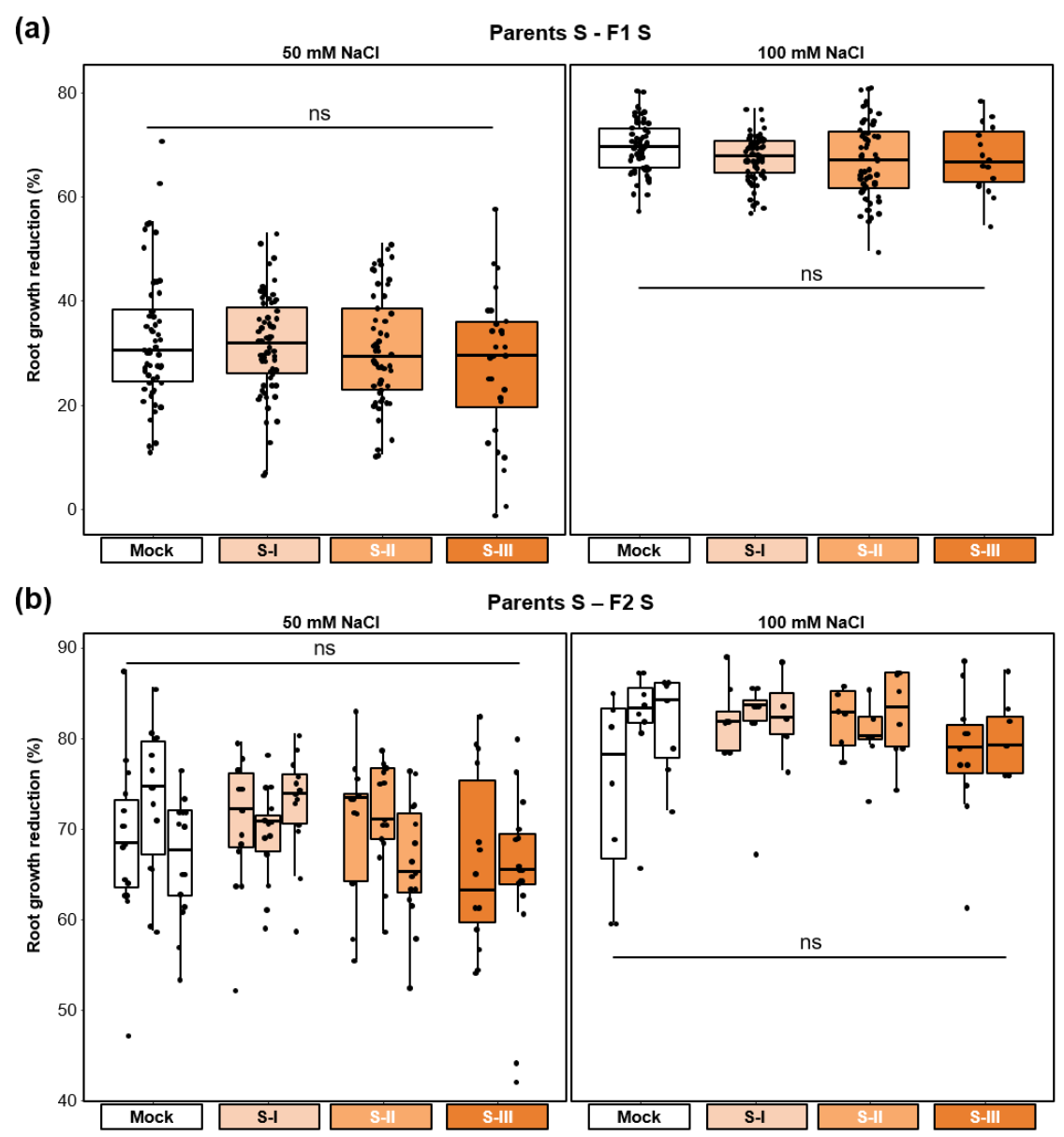

Fig. 5. Lack of salt-elicited TAR in matched environments. Parental plants had been exposed to different stress intensities by soil salinity (NaCl; Mock, S-I, S-II, S-III). Salt tolerance of F1 and F2 plants was quantified by root growth reduction (\%) over a 5-day period on $\mathrm{NaCl}$-containing agar medium relative to the average root growth on agar medium without $\mathrm{NaCl}$. Boxplots show the interquartile range (IQR; box) $\pm 1.5 \times$ IQR (whiskers), including median (horizonal line) and replication units (dots). (a) Unaltered tolerance of $\mathrm{F} 1$ plants to 50 and $100 \mathrm{mM} \mathrm{NaCl}$. Data represent growth reduction percentages of single plants within $\mathrm{F} 1$ populations from similarly treated parents $(n=60)$. ns: no statistically significant differences (ANOVA; $\alpha=0.05$ ). Root growth data for individual $\mathrm{F} 1$ populations are shown in Fig. S4a; root tolerance data for individual F1 populations are shown in Fig. S4c. (b) Unaltered tolerance of F2 plants to 50 and 100 $\mathrm{mM} \mathrm{NaCl}$ after one stress-free F1 generation. Data represent growth reduction percentages of single plants within F2 populations that share a common parental ancestor $(n=18-20)$. ns: no statistically significant differences (ANOVA; $\alpha=0.05$ ). Root growth data for individual F2 populations are shown in Fig. S4b; root tolerance data for individual F2 populations are shown in Fig. S4d.

Figure 5: This is a caption

\section{TAR is associated with costs that become apparent in mismatched environments.}

To investigate the resistance phenotypes of our F1 populations against stresses other than the parental stress (mismatched environments), we used a reciprocal experimental design based on the three parental stress treatments (Fig. 1). We have previously reported that Pst-elicited TAR is associated with increased susceptibility to the necrotrophic fungus A. brassicicola (Luna et al., 2012). In agreement with this finding, 
F1 progeny from Pst-infected plants developed larger lesions after inoculation with the necrotrophic Pc (Fig. 6a and Fig. S5a). Furthermore, F1 progeny from Pst-exposed parents showed a statistically significant increase in root growth inhibition by $50 \mathrm{mM} \mathrm{NaCl}$ (Fig. 6b and Fig. S5b,c), indicating increased sensitivity to salt stress. Next, we investigated F1 progeny from $P c$-infected parents for resistance against biotrophic $H p a$ and salt stress. F1 populations from parents exposed to the two highest severities of $P c$ disease showed increased susceptibility to $\mathrm{Hpa}$ (Fig. 6c and Fig. S6a) but were unaffected in salt tolerance (Fig. 6d and Fig. S6b,c). Together, these results indicate that the potential benefits of pathogen-induced TAR are traded off against costs of increased susceptibility to other stresses that become apparent in mismatched environments.
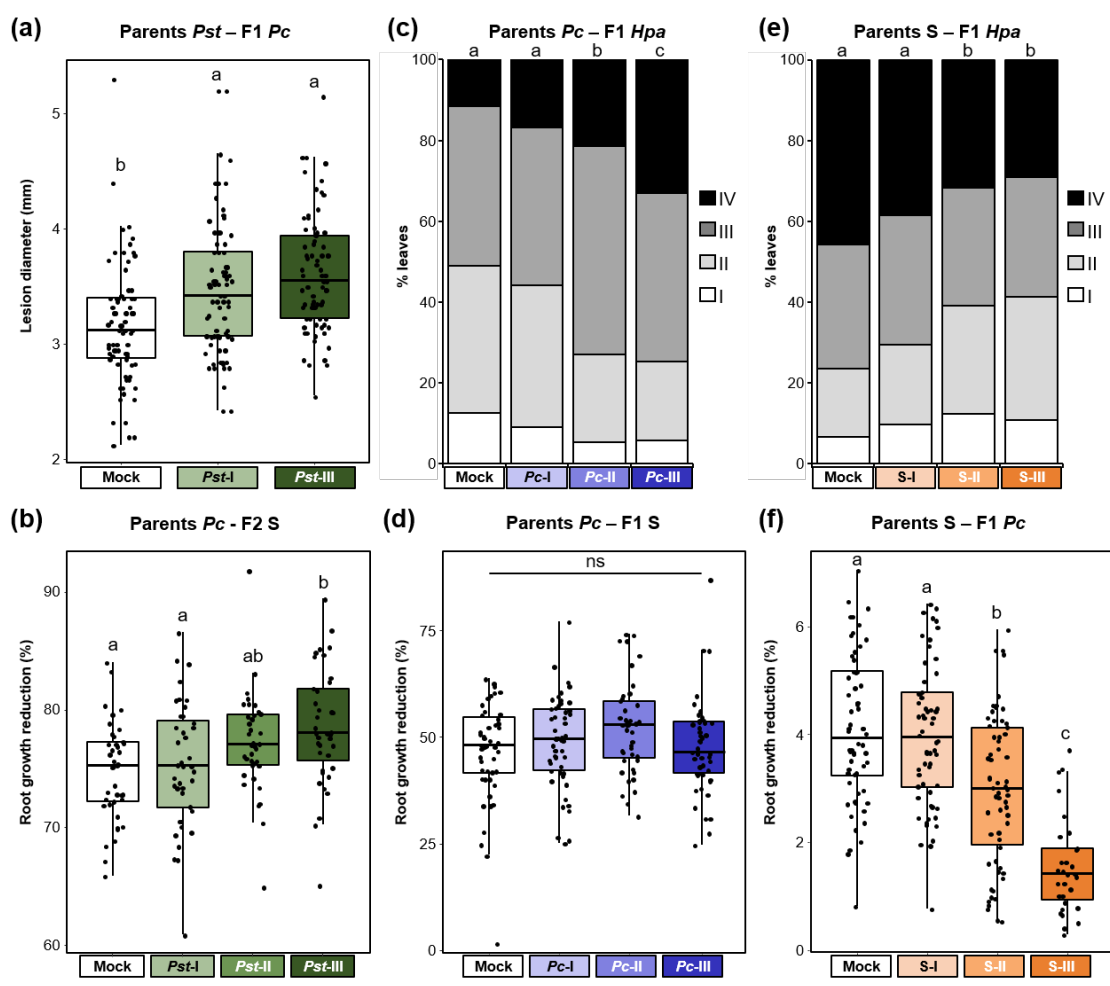

(d)

(f)
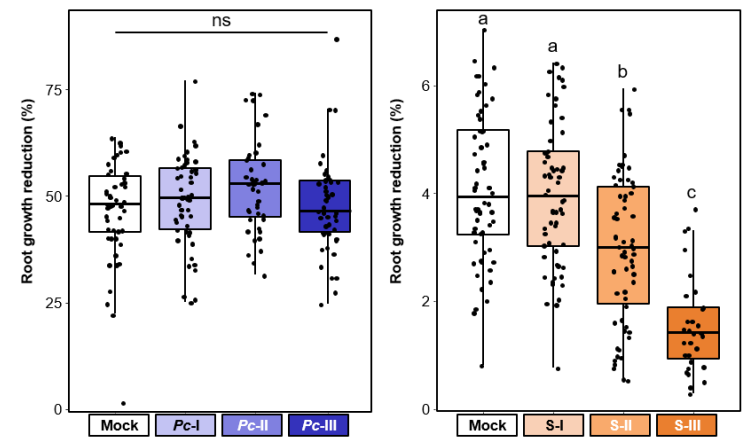

Fig. 6. Costs and benefits of TAR in mismatched environments. Parental plants had been exposed to different stress severities by Pst (green) $P c$ (blue) or soil salinity (orange). F1 plants were tested for resistance against different stresses than the parental stress. (a) Increased $P c$ susceptibility in F1 progeny from Pst-exposed parents. Box plots show lesion diameters ( $\mathrm{mm}$ ) of plants within $\mathrm{F} 1$ populations from similarly treated parental plants ( $n=76-80$ ). See legend of Fig. 4 for details. Different letters indicate statistically significant differences between parental treatments (ANOVA + Tukey post-hoc test; $\alpha=0.05$ ). Data for individual F1 populations are shown in Fig. S5a. (b) Reduced salt tolerance in F1 progeny from Pst-exposed parents. Box plots show root growth reduction percentages by $50 \mathrm{mM} \mathrm{NaCl}$ of plants within $\mathrm{F} 1$ populations from similarly treated
parental plants $(n=40$ ) See legend of Fig. 5 for details. Different letters indicate statistically significant differences (ANOVA + Tukey post-hoc test

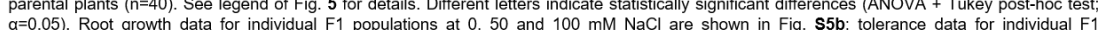
populations to 50 and $100 \mathrm{mM}$ NaCl are shown in Fig. S5c. (c) Increased Hpa susceptibility in F1 progeny from Pc-exposed parents. Stacked bars

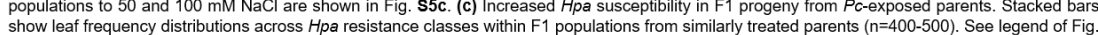

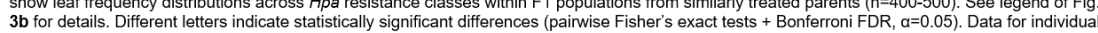
F1 populations are shown in Fig. S6a. (d) Unaltered salt tolerance in F1 progeny from Pc-exposed parents. Box plots show root growth reduction percentages by $50 \mathrm{mM} \mathrm{NaCl}$ of plants within F1 populations from similarly treated parental plants (n=47). See legend of Fig. 5 for details. ns: no statistically significant differences (ANOVA; $\mathrm{a}=0.05$ ). Root growth data for individual $\mathrm{F} 1$ populations at 0,50 and $100 \mathrm{mM} \mathrm{NaCl}$ are shown in Fig. S6B; tolerance data for individual F1 populations to 50 and $100 \mathrm{mM}$ NaCl are shown in Fig. S6c. (e) Non-specific TAR against Hpa in F1 progeny from NaCl-exposed parents. Stacked bars show leaf frequency distributions across Hpa resistance classes within F1 populations from similarly treated parents $(n=350-800)$. See legend of Fig. $3 b$ for details. Different letters indicate statistically significant differences (Pairwise Fisher's exac tests + Bonferroni $F \mathrm{FR}, \mathrm{a}=0.05$ ). Data for individual $\mathrm{F} 1$ populations are shown in Fig. S7a. (f) Non-specific TAR against $P c$ in $\mathrm{F} 1$ progeny from NaCltreated parents. Box plots show lesion diameters $(\mathrm{mm})$ of plants within $\mathrm{F} 1$ populations from similarly treated parental plants $(\mathrm{n}=30-60)$. See legend Data for individual F1 populations are shown in Fig S S7b.

Figure 6: This is a caption

Surprisingly, F1 progeny from parents exposed to the highest degrees of soil salinity showed increased resistance to both biotrophic $H p a$ and necrotrophic $P c$ (Fig. 6e,f and Fig. S7a,b). This finding argues against 
the concept of TAR specificity, since the benefits, rather than the costs, were apparent in mismatched environments. However, the evolutionary significance of this non-specific TAR by salt stress must be considered against the lack of adaptive effects in the matched environment (Fig. 5) and the severe fitness costs arising from reduced plant growth, seed production and seed viability (Fig. 2).

\section{Stress intensity acts as a weighted indicator for TAR investment.}

Since TAR is associated with costs in mismatched environments, we considered the possibility that plants can adjust TAR investment in accordance to the reliability of the environmental stress signal. We hypothesised that severe stress is perceived as a more reliable predictor of the progeny environment, resulting in stronger TAR investment. In matched environments, Pst- and Pc-elicited TAR was strongest in F1 progeny from parents exposed to the highest stress levels, although the difference in TAR intensity between the lowest and highest parental stress intensities was not statistically significant (Fig. 3a,b, Fig. S2a,b, Fig. 4a and Fig. S3a). Similarly, in mismatched environments, F1 progeny from parents exposed to the highest level of $P s t$ disease showed increased $P c$ susceptibility compared to progeny from parents exposed to the lowest level of Pst disease; however, this difference was not statistically significant (Fig. 6a and Fig. S5a). By contrast, the increased sensitivity of F1 progeny from Pst-exposed parents to salt showed statistically significant differences that were proportional to the levels of parental disease severity (Fig. 6b and Fig. S5c). Similarly, Hpa susceptibility in F1 progeny from $P c$-exposed parents (Fig. 6c and Fig. S6a), as well as nonspecific TAR in F1 progeny from salt-exposed parents, showed statistically significant differences that were proportional to the level of parental stress (Fig. 6e,f, Fig. S7). Thus, although the intensity of pathogenelicited TAR in matched environments of F1 progeny does not show a statistically significant dose effect, the response is associated with dose-dependent costs that become evident in mismatched environments. Finally, we investigated whether the transgenerational stability of TAR into the F2 generation is proportional to the level of parental stress in matched environments. To this end, we determined pathogen-elicited TAR in F2 progeny after one stress-free F1 generation. In contrast to F1 progeny (Fig. 3b and Fig. S2b), F2 progeny from parents exposed to the lowest levels of Pst disease no longer showed TAR against Hpa (Fig. 3c and Fig. S2c). Furthermore, only one F2 population from parents exposed to intermediate levels of Pst disease had maintained a statistically significant TAR response, whereas all F2 populations from parents exposed to the highest levels of Pst disease had maintained a statistically significant TAR response (Fig. 3c and Fig. S2c). F2 populations from parents exposed to low and intermediate levels of $P c$ disease all failed to show TAR (Fig. 4b and Fig. S3b). However, ANOVA of pooled F2 populations from similarly treated parental plants, as well as nested ANOVA with F2 population as a random variable, revealed a statistically significant effect of parental stress treatment (Fig. S3b), indicating a residual amount of TAR in F2 populations from $P c$-exposed parents. This is further supported by the observation that F2 populations from parents exposed to the highest degree of $P c$ disease also showed the highest level of $P c$ resistance (Fig. $\mathbf{4 b}$ and Fig. S3b). Hence, TAR in response to relatively high levels of disease by $P s t$ or $P c$ can persist into the F2 generation, whereas TAR elicited by low or intermediate disease levels is reverted or weakened after one stress-free F1 generation. Together, these results demonstrate that the intensity, costs and/or transgenerational stability of TAR have a dose-dependent relationship with parental stress intensity, which supports our hypothesis that plants use stress intensity as a weighted indicator of TAR investment.

\section{Discussion}

Evolutionary models predict that parental effects on specific traits act as an adaptive mechanism to increase fitness in changeable environments (Leimar \& McNamara, 2015; Pigeault et al., 2016; Proulx \& Teotonio, 2017). However, despite numerous reports of transgenerational effects of stress in plants, it has remained 
uncertain whether these responses are adaptive (Uller et al., 2013; Burggren, 2015; Crisp et al., 2016). Although the importance of full factorial designs to address this question has been stressed (Marshall \& Uller, 2007; Bonduriansky et al., 2012; Uller et al., 2013; Burgess \& Marshall, 2014; Tetreau et al., 2019),

Most previous reports about TAR are based on experiments in which resistance was tested in matched environments and therefore do not address the specificity of the response. The reciprocal design of our study (Fig. 1) allowed us to examine progeny resistance phenotypes in both matched and mismatched environments. In the case of disease stress, we found strong evidence that TAR is specific. Disease by biotrophic $P s t$ bacteria elicits TAR against taxonomically unrelated $H p a$ with a similar biotrophic lifestyle (Fig. 3b,c) but fails to protect against necrotrophic $P c$ or abiotic salt stress (Fig. 6a,b,c). Similarly, parental disease by $P c$ elicited TAR against the same necrotrophic fungus (Fig. 4) but not against biotrophic Hpa and abiotic salt stress (Fig. 6c,d). This specificity supports the notion that pathogen-elicited TAR is an adaptive response. By contrast, we found no evidence for increased salt tolerance in F1 or F2 progeny from salt-exposed parents (Fig. 5), even though F1 progeny from salt-exposed parents showed non-specific resistance against biotrophic and necrotrophic pathogens (Fig. 6e,f). Previous studies have shown that abiotic stress causes non-specific transgenerational tolerance to other abiotic stresses (Boyko et al., 2010; Rahavi et al., 2011), but not biotic stresses. The lack of specific TAR against salt stress (Fig. 4), along with the associated reduction in reproductive fitness (Fig. 2b,c), suggests that salt-elicited TAR is non-adaptive and unlikely provides a selective advantage in natural environment. The discrepancy between salt- and pathogen-elicited TAR can be explained by differences in parental response to these stresses (Fig. 2a,b,c). While all three stresses caused an immediate reduction in plant growth, Pst and $P c$ had no, or even stimulatory, effects on reproductive fitness (Fig. 2a,b,c), indicating that Arabidopsis uses induced resistance to mitigate Pst and $P c$ stress, compensating potential fitness loss from reduced growth with increased seed production at the end of its life cycle. This ability to recover from stress constitutes a reliable cue that TAR will improve fitness of progeny in the same environment, thereby justifying TAR investment. By contrast, the progressive loss of seed production and viability upon increasing levels of stress from soil salinity (Fig. $\mathbf{2 b}$, c) indicates that the parental plants do not recover well from this stress. Investment in a transgenerational response is therefore not beneficial, even in a matched environment. This hypothesis is supported by modelling which shows that TAR in invertebrates occurs at intermediate levels of disease stress, but not when there are more severe impacts on mortality (Pigeault et al., 2016).

Costs are central to the evolution of adaptive transgenerational responses. The fact that pathogenelicited TAR is inducible and reversible in the absence of stress implies that the response is associated with costs (Fig. 3,4) (Stassen et al., 2018). Previous work has identified transgenerational impacts of parental stress on vegetative and reproductive development (e.g. Rahavi et al., 2011; Suter \& Widmer, 2013; Groot et al., 2016), but it remains unclear how far these changes influence fitness. While we did not observe consistent effects on plant growth or seed set in F1 and F2 progeny from disease-exposed plants (data not shown), the reciprocal design of our experiments strongly indicates ecological costs arising from increased susceptibility to other stresses (Fig. 6a,b,c,d). Antagonism between plant defence pathways against biotrophic pathogens, necrotrophic pathogens and abiotic stress is well-documented (Koornneef \& Pieterse, 2008; Pieterse et al., 2012), and transgenerational persistence of these effects have been reported previously (Luna et al., 2012; Singh et al., 2017). Accordingly, we propose that negative cross-talk between defence pathways imposes a major cost on adaptive TAR responses to pathogens.

Although examples of transgenerational phenotypic plasticity are now widespread, there are instances where researchers have failed to identify such effects in Arabidopsis (Pecinka et al., 2009; Suter \& Widmer, 2013). Indeed, evolutionary theory predicts that transgenerational plasticity is not a universal trait and that its occurrence depends highly on genotype, mode of reproduction, ecological niche and life history traits, as well as the nature and consistency of the eliciting stress (Crisp et al., 2016; Groot et al., 2016). Compared to fixed genetic adaptation, it can be expected that transgenerational phenotypic plasticity offers a suitable adaptation strategy under variable environments. However, it is unlikely that either adaptation strategy will be under positive selection in highly variable, unpredictable environments, since the frequency of incurred costs would outweigh the specific benefits. Adaptive parental effects would therefore more likely emerge 
when the same type of environmental stress occurs regularly (Tricker, 2015). Under such conditions, stressexposed plants can optimise fitness either by maximising their own immediate performance to the detriment of their progeny ('selfish parental effects'), or by modifying progeny traits to provide enhanced performance in the altered environment (Marshall \& Uller, 2007). The latter strategy can take form in either a diversified bet-hedging strategy, or a more deterministic provision of specific adaptive traits, such as pathogen-elicited TAR, which is tailored to the parental environment (Marshall \& Uller, 2007; Crean \& Marshall, 2009; Proulx \& Teotonio, 2017). Not only do evolutionary models predict that transgenerational phenotypic plasticity is likely to evolve in fluctuating environments (Leimar \& McNamara, 2015; Pigeault et al., 2016; Proulx \& Teotonio, 2017), the model developed by Proulx and Teotonio (2017) suggests that deterministic (adaptive) parental effects like pathogen-elicited TAR provide increased fitness over a wider range of environmental parameters than a randomising bet-hedging strategy.

Central to the provision of adaptive transgenerational traits is the ability to make accurate and reliable predictions about future progeny environments. While this aspect has been emphasised in both evolutionary theory and modelling of parental effects (Burgess \& Marshall, 2014; Leimar \& McNamara, 2015), it has rarely been addressed experimentally. The few studies to have included this concept applied the same stress repeatedly over multiple generations rather than applying different stress intensities within the same generation. In one of the most comprehensive studies of this type, Groot et al. (2016) found complex interactions between parental (P), grandparental (GP) and great-grandparental (GGP) salt stress in Arabidopsis. When the stress was applied to only one generation, $\mathrm{P}$ effects were typically stronger than GP and GGP effects. For treatments over multiple generations, the impacts of GP and GGP stress were additive to P treatments for some traits, but antagonistic for others. Furthermore, the transgenerational effects in the study by Groot et al. (2016) varied between controlled environments and field conditions, making it difficult to conclude whether the effects were adaptive. In our study, varying levels of three different stresses were applied within one generation, providing a straightforward design to assess whether parents can distinguish stress severities and adjust the transgenerational response accordingly. Our pathogen treatments resulted in dose-dependent impacts on relative growth rate during the treatment period (Fig. 2a), indicating that Arabidopsis perceives these stresses in a dose-dependent manner. Furthermore, analysis of the transgenerational stability of TAR provided evidence for a dose-dependent relationship with parental disease severity. Although F1 populations from both $P$ st- and $P c$-exposed parents expressed TAR to statistically similar levels across stress levels (Fig. 3a,b and Fig. 4a), TAR only persisted over a stress-free generation when elicited by the highest stress levels (Fig. 3c and Fig. 4b). Furthermore, in mismatched environments, there was a dose-dependent effect on the costs of pathogen-elicited TAR: both salt sensitivity of F1 progeny from Pst-infected parents and Hpa susceptibility of F1 progeny from $P c$-infected parents correlated with the severity of parental disease stress treatment (Fig. 6b,c). Overall, these results support our hypothesis that plants perceive disease severity as a predictive cue to adjust TAR investment.

Collectively, our study demonstrates that parental investment in pathogen-elicited TAR provides fitness benefits in matched environments and costs in mismatched environments. This stress-specific TAR is dependent on the intensity of the stress experienced by the parents, which holds predictive value for future progeny environments. Accordingly, our findings are consistent with the evolutionary prediction that pathogen-elicited TAR is a genuine adaptive trait in Arabidopsis. In one of the most convincing cases of adaptive parental effects in plants, Galloway and Etterson (2007) used field-based studies to demonstrate adaptive transgenerational plasticity in response to the light environment. It will now be of interest to undertake ecological field studies and verify our laboratory experiments in support of TAR as an adaptive transgenerational effect in nature. 


\section{Materials and Methods}

\section{Plant material and growth conditions.}

All Arabidopsis thaliana lines described in this study are in the genetic background of accession Col-0 (NCBI, Tax ID 3702). To exclude confounding effects of TAR from stress in previous generations, all lines originated from a common ancestor of a population that had maintained under stress-free conditions (mock-inoculated) in two previous generations (Luna et al., 2012). Except for the stress treatments, all plants were grown under similar conditions (see Supplementary Methods in Supporting Information for details). To generate F1 populations, 6-8 parental plants of 4.5-weeks-old were subjected to mock/stress treatments over a duration of 3 weeks, after which 4 parental plants with representative symptoms were moved to long-day conditions (16 h light/8 h darkness) to set seed and generate F1 populations (Fig. 1). Three individual plants from each F1 population were kept apart under stress-free conditions to set seed, resulting in $3 \mathrm{~F} 2$ populations from each F1 population and a total of 12 F2 populations per parental treatment (Fig. 1). Details of all F1 and F2 populations are presented in Table S1 in the Supporting Information.

\section{Stress treatments of parental plants.}

Inoculation with biotrophic Pseudomonas syringae pv. tomato (Pst) was performed at 3-4 day intervals over a total period of 3 weeks, as detailed in the Supplementary Methods. Plants were subjected to different Pst disease pressures: no disease (Mock; 6 subsequent inoculations with the mock suspension), low disease (Pst-I; 2 inoculations with Pst followed by 4 mock inoculations), medium disease (Pst-II; 4 inoculations with $P$ st followed by 2 mock inoculations solution), and high disease (Pst-III; 6 subsequent inoculations with Pst). To ensure necrotrophic infection by Plectophaerella cucumerina $(P c)$, inoculation was performed by placing $6 \mu \mathrm{l}$-droplets $\left(10^{6}\right.$ spores $\left.\mathrm{ml}^{-1}\right)$ onto fully expanded leaves of approximate similar age (Petriacq et al., 2016), as detailed in the Supplementary Methods. Plants were subjected to different $P c$ disease pressures: no disease (Mock; 6 leaves were mock-inoculated), low disease ( $P c$-I; 2 leaves $P c$-inoculated and 4 leaves mock-inoculated), medium disease ( $P c$-II; 4 leaves $P c$-inoculated and 2 leaves mock-inoculated), and high disease ( $P c$-III; 6 leaves $P c$-inoculated). After inoculation, plants were kept at $100 \% \mathrm{RH}$ for 2 weeks until visible disease symptoms appeared in $>80 \%$ of the leaf surface (necrosis and chlorosis). To prevent sporulation and ongoing disease progression, plants were returned to $60 \% \mathrm{RH}$ before moving to long-day conditions 1 week later. Salt stress was applied by soil-drenching with $100 \mathrm{mM} \mathrm{NaCl}$ solution. Plants were subjected to different stress levels over the 3-week period: mock treatment (S-I; drenched 6x with water), low stress (S-II; drenched $2 \mathrm{x}$ with $\mathrm{NaCl}$ and $4 \mathrm{x}$ with water), medium stress (S-III; drenched $4 \mathrm{x}$ with $\mathrm{NaCl}$ and $2 \mathrm{x}$ with water), high stress (S-III; plants drenched $6 \mathrm{x}$ with $\mathrm{NaCl}$ ). Plants returned to a normal watering regime when moved to long-day conditions.

\section{Quantification of fitness parameters.}

Relative growth rate (RGR) was determined non-destructively by quantification of green leaf area (GLA) before and after stress treatments, as detailed in the Supplementary Methods. Reproductive fitness was estimated by seed production and seed viability, as described in the Supplementary Methods. 


\section{Quantification of transgenerational resistance phenotypes.}

To quantify resistance against biotrophic $P s t$, the inoculum was prepared and adjusted to $2 \times 10^{5} \mathrm{CFU} \mathrm{mL}^{-1}$ (see Supplementary Methods). Inoculation was performed by syringe infiltration of 4 leaves/plant of approximate similar age. Bacterial growth was quantified at 3 days post inoculation (dpi) by dilution plating on selective agar plates (see Supplementary Methods). Inoculation with biotrophic Hpa and quantification of Hpa resistance was performed as described previously (Lopez Sanchez et al., 2016; see also Supplementary Methods). Quantification of salt tolerance was based on root growth analysis on agar plates containing $0 \mathrm{mM}$, $50 \mathrm{mM}$ and $100 \mathrm{mM} \mathrm{NaCl}$. Assays were conducted as described previously (Verslues et al., 2006; Claeys et al., 2014) with minor modifications (see Supplementary Methods).

\section{Statistical analysis.}

Analytical statistics was performed using R studio (v 1.1.456, https://rstudio.com/), supporting R software (v 3.5.1, https://www.r-project.org/). Statistical significance of treatment effects on continuous variables was analysed by linear models; statistical significance of treatment effects on categorical variables (class frequencies) was analysed by Fisher's exact tests. Details about data transformations, statistical models, and R software packages are described in the Supplementary Methods.

\section{Acknowledgements}

The work was supported by ERC (no. 309944 "Prime-A-Plant" and no. 824985 "ChemPrime") to JT, a Research Leadership Award from the Leverhulme Trust (no. RL-2012-042) to JT, a BBSRC-IPA grant to JT (BB/P006698/1) and a BBSRC responsive mode grant to MR and JT (BB/L008939/1). Seeds of Arabidopsis F1 and F2 populations can be made available upon request with JT (Table S1).

\section{Competing interests statement}

There are no competing interests of a financial or non-financial nature.

\section{Author Contributions}

JT and MR conceived the project; JT and ALS designed and supervised the experiments; ALS, DP and LF performed bioassays; JT performed statistical analyses; MR, ALS and JT wrote the manuscript. All authors reviewed and approved the final manuscript. All authors declare no competing interests. 


\section{References}

Bonduriansky R, Crean AJ, Day T. 2012. The implications of nongenetic inheritance for evolution in changing environments. Evolutionary Applications 5(2): 192-201.

Bošković A, Rando OJ. 2018. Transgenerational epigenetic inheritance. Annual Review of Genetics 52(1): 21-41.

Boyko A, Blevins T, Yao Y, Golubov A, Bilichak A, Ilnytskyy Y, Hollander J, Meins F, Kovalchuk I. 2010. Transgenerational adaptation of Arabidopsis to stress requires DNA methylation and the function of Dicer-Like proteins. PLoS ONE 5(3): e9514.

Burgess SC, Marshall DJ. 2014. Adaptive parental effects: the importance of estimating environmental predictability and offspring fitness appropriately. Oikos 123(7): 769-776.

Burggren WW. 2015. Dynamics of epigenetic phenomena: intergenerational and intragenerational phenotype 'washout'. The Journal of Experimental Biology 218(1): 80-87.

Claeys H, Van Landeghem S, Dubois M, Maleux K, Inze D. 2014. What is stress? Dose-response effects in commonly used in vitro stress assays. Plant Physiol 165(2): 519-527.

Crean AJ, Marshall DJ. 2009. Coping with environmental uncertainty: dynamic bet hedging as a maternal effect. Philosophical Transactions of the Royal Society B: Biological Sciences 364(1520): 10871096.

Crisp PA, Ganguly D, Eichten SR, Borevitz JO, Pogson BJ. 2016. Reconsidering plant memory: Intersections between stress recovery, RNA turnover, and epigenetics. Sci Adv 2(2): e1501340.

Furci L, Jain R, Stassen J, Berkowitz O, Whelan J, Roquis D, Baillet V, Colot V, Johannes F, Ton J. 2019. Identification and characterisation of hypomethylated DNA loci controlling quantitative resistance in Arabidopsis. eLife 8: e40655.

Galloway LF, Etterson JR. 2007. Transgenerational plasticity is adaptive in the wild. Science 318(5853): 1134-1136.

Gehring M. 2019. Epigenetic dynamics during flowering plant reproduction: evidence for reprogramming? New Phytologist 224(1): 91-96.

Groot MP, Kooke R, Knoben N, Vergeer P, Keurentjes JJB, Ouborg NJ, Verhoeven KJF. 2016. Effects of multi-generational stress exposure and offspring environment on the expression and persistence of transgenerational effects in Arabidopsis thaliana. PLoS ONE 11(3): e0151566.

Herman JJ, Sultan SE. 2011. Adaptive transgenerational plasticity in plants: case studies, mechanisms, and implications for natural populations. Front Plant Sci 2: 102.

Herman JJ, Sultan SE, Horgan-Kobelski T, Riggs C. 2012. Adaptive transgenerational plasticity in an annual plant: grandparental and parental drought stress enhance performance of seedlings in dry soil. Integr Comp Biol 52(1): 77-88.

Holeski LM, Jander G, Agrawal AA. 2012. Transgenerational defense induction and epigenetic inheritance in plants. Trends Ecol Evol 27(11): 618-626.

Iwasaki M, Paszkowski J. 2014. Identification of genes preventing transgenerational transmission of stress-induced epigenetic states. Proc Natl Acad Sci U S A 111(23): 8547-8552.

Koornneef A, Pieterse CM. 2008. Cross talk in defense signaling. Plant Physiol 146(3): 839-844.

Lämke J, Bäurle I. 2017. Epigenetic and chromatin-based mechanisms in environmental stress adaptation and stress memory in plants. Genome Biology 18(1): 124. 
Leimar O, McNamara JM. 2015. The evolution of transgenerational integration of information in heterogeneous environments. The American Naturalist 185(3): E55-E69.

Lopez Sanchez A, Stassen JHM, Furci L, Smith LM, Ton J. 2016. The role of DNA (de)methylation in immune responsiveness of Arabidopsis. Plant Journal 88(3): 361-374.

Luna E, Bruce TJA, Roberts MR, Flors V, Ton J. 2012. Next-generation systemic acquired resistance. Plant Physiology 158(2): 844-853.

Luna E, Ton J. 2012. The epigenetic machinery controlling transgenerational systemic acquired resistance. Plant Signal Behav 7(6): 615-618.

Marshall DJ, Uller T. 2007. When is a maternal effect adaptive? Oikos 116(12): 1957-1963.

Pecinka A, Rosa M, Schikora A, Berlinger M, Hirt H, Luschnig C, Mittelsten Scheid O. 2009. Transgenerational stress memory is not a general response in Arabidopsis. PLoS ONE 4(4): e5202.

Perez MF, Lehner B. 2019. Intergenerational and transgenerational epigenetic inheritance in animals. Nature Cell Biology 21(2): 143-151.

Petriacq P, Stassen JH, Ton J. 2016. Spore Density Determines Infection Strategy by the Plant Pathogenic Fungus Plectosphaerella cucumerina. Plant Physiol 170(4): 2325-2339.

Pieterse CM, Van der Does D, Zamioudis C, Leon-Reyes A, Van Wees SC. 2012. Hormonal modulation of plant immunity. Annu Rev Cell Dev Biol 28: 489-521.

Pigeault R, Garnier R, Rivero A, Gandon S. 2016. Evolution of transgenerational immunity in invertebrates. Proceedings of the Royal Society B: Biological Sciences 283(1839).

Proulx SR, Teotonio H. 2017. What kind of maternal effects can be selected for in fluctuating environments? Am Nat 189(6): E118-e137.

Rahavi MR, Migicovsky Z, Titov V, Kovalchuk I. 2011. Transgenerational adaptation to heavy metal salts in Arabidopsis. Frontiers in Plant Science 2(91).

Rasmann S, De Vos M, Casteel CL, Tian D, Halitschke R, Sun JY, Agrawal AA, Felton GW, Jander G. 2012. Herbivory in the previous generation primes plants for enhanced insect resistance. Plant Physiol 158(2): 854-863.

Schlichting CD. 1986. The evolution of phenotypic plasticity in plants. Annual Review of Ecology and Systematics 17(1): 667-693.

Singh P, Dave A, Vaistij FE, Worrall D, Holroyd GH, Wells JG, Kaminski F, Graham IA, Roberts MR. 2017. Jasmonic acid-dependent regulation of seed dormancy following maternal herbivory in Arabidopsis. New Phytologist 214(4): 1702-1711.

Stassen JHM, Lopez A, Jain R, Pascual-Pardo D, Luna E, Smith LM, Ton J. 2018. The relationship between transgenerational acquired resistance and global DNA methylation in Arabidopsis. Sci Rep 8(1): 14761.

Suter L, Widmer A. 2013. Phenotypic effects of salt and heat stress over three generations in Arabidopsis thaliana. PLoS ONE 8(11): e80819.

Tetreau G, Dhinaut J, Gourbal B, Moret Y. 2019. Trans-generational immune priming in invertebrates: Current knowledge and future prospects. Frontiers in Immunology 10(1938).

Tricker PJ. 2015. Transgenerational inheritance or resetting of stress-induced epigenetic modifications: two sides of the same coin. Front Plant Sci 6: 699.

Uller T, Nakagawa S, English S. 2013. Weak evidence for anticipatory parental effects in plants and animals. J Evol Biol 26(10): 2161-2170. 
Verslues PE, Agarwal M, Katiyar-Agarwal S, Zhu J, Zhu JK. 2006. Methods and concepts in quantifying resistance to drought, salt and freezing, abiotic stresses that affect plant water status. Plant $J$ 45(4): 523-539.

Wilkinson SW, Magerøy MH, López Sánchez A, Smith LM, Furci L, Cotton TEA, Krokene P, Ton J. 2019. Surviving in a hostile world: Plant strategies to resist pests and diseases. Annual Review of Phytopathology 57(1): 505-529.

\section{Figure legends}

Fig. 1. Full factorial experimental design of the study. Arabidopsis thaliana plants (accession Col0 ) from a common ancestor were exposed to increasing stress intensities (Mock, Low, Medium and High) by the (hemi)biotrophic bacterial pathogen Pseudomonas syringae pv. tomato (green), the necrotrophic fungal pathogen Plectosphaerella cucumerina (blue), or soil salinity $(\mathrm{NaCl}$; orange). Plants in this parental generation $(\mathrm{P})$ were evaluated for impacts on fitness parameters. Four plants per stress level were selected to generate $\mathrm{F} 1$ populations, which were analysed for transgenerational changes in resistance against all 3 stresses, in order to determine the specificity of transgenerational acquired resistance (TAR), potential costs arising from increased susceptibility, and dose-dependency of TAR intensity on parental stress. Four individual plants from 3 independent F1 populations were randomly selected to set seed in the absence of stress. The resulting F2 populations were analysed for resistance against the parental stress to examine dose-dependent effects on TAR stability. Circles indicate individual plants; small (thin-lined) boxes indicate F1/F2 populations derived from a common ancestor in the previous generation; big (bold-lined) boxes indicate pooled F1/F2 populations from a common ancestor 2 generations earlier.

Fig. 2. Differential impacts of three (a)biotic stresses on parental fitness parameters. Plants in the parental generation (4.5-weeks-old) were exposed to varying stress intensities by $P$. syringae pv tomato (Mock, Pst-I, Pst-II, Pst-III), P. cucumerina (Mock, Pc-I, Pc-II, Pc-III) or soil salinity (Mock, S-I, S-II, S-III) over a 3 -week period before transferring to long-day conditions to trigger flowering and set seed. Boxplots show the interquartile range (IQR; box) $\pm 1.5 x I Q R$ (whiskers), including median (horizonal line) and replication units (single dots). (a) Impacts on relative growth rate (RGR) during the period of stress exposure. Data represent RGR values of single plants $(n=5-6)$ normalised to the average RGR of Mock-treated plants (100\%). Different letters indicate statistically significant differences (ANOVA + Tukey post-hoc test, $\alpha=0.05)$. (b) Impacts on seed production. Data represent seed numbers per plant $(\mathrm{n}=5-6)$ normalised to average value of Mock-treated plants (100\%). Different letters indicate statistically significant differences (Pst: Welch ANOVA + Games-Howell posthoc test, $\alpha=0.05 ; P c$ and salt: ANOVA + Tukey post-hoc test, $\alpha=0.05$ ). (c) Impacts on seed viability. Seed viability was determined 5 days after planting of surface-sterilised and stratified seeds onto 0.2x Murashige and Skoog (MS) agar plates. Data represent mean germination percentages per plate $(25$ seeds/plate) of seed batches from 4 similarly treated parents $(\mathrm{n}=15-60)$. Different letters indicate statistically significant differences (Welch ANOVA + Games-Howell post-hoc test; $\alpha=0.05)$. Viability data for seed batches from individual plants are presented in Fig. S1a-c.

Fig. 3. Parental plants had been exposed to different disease severities by the biotrophic bacterium Pseudomonas syringae pv. tomato (Pst; Mock, Pst-I, Pst-II, Pst-III).

F1 and F2 plants were analysed for resistance against the same pathogen (Pst) and/or the biotrophic 
Oomycete Hyaloperonospora arabidopsidis ( $\mathrm{Hpa}$ ). (a) TAR against Pst in F1 progeny at 3 days post inoculation (dpi). Boxplots show the interquartile range (IQR; box) $\pm 1.5 x I Q R$ (whiskers), including median (horizonal line) and replication units (dots). Data represent ${ }^{10} \mathrm{Log}$-transformed bacterial titres $\left(\mathrm{Log} \mathrm{cfu} \mathrm{cm}{ }^{-2}\right)$ in leaves of single plants within F1 populations from similarly treated parents $(n=42)$. Different letters indicate statistically significant differences (Welch ANOVA + Games-Howell test, $\alpha=0.05$ ). Data for individual F1 populations are shown in Fig. S2a. (b) TAR against Hpa in F1 progeny. Hpa colonisation was quantified at 6 dpi by assigning trypan-blue stained leaves to $4 \mathrm{Hpa}$ resistance classes (I: healthy; II: hyphal colonisation only; III hyphal colonization with conidiospores; IV hyphal colonisation with conidiospores and oospores). Stacked bars show leaf frequency distributions within F1 populations from similarly treated parental plants ( $\mathrm{n}=600-1000)$. Different letters indicate statistically significant differences (Pairwise Fisher's exact tests + Bonferroni FDR, $\alpha=0.05$ ). Data for individual F1 populations are shown in Fig. S2b. (c) TAR against Hpa in F2 progeny at 6 dpi after one stress-free F1 generation. Stacked bars show leaf frequency distributions across Hpa resistance classes within F2 populations that share a common parental ancestor $(\mathrm{n}=300-350)$. Different letters indicate statistically significant differences (Pairwise Fisher's exact tests + Bonferroni FDR; $\alpha=0.05)$. Data for individual F2 populations are shown in Fig. S2c.

Fig. 4. Intensity and transgenerational stability of Plectosphaerella cucumerina-elicited TAR in matched environments. Parental plants had been exposed to different disease severities by necrotrophic Plectosphaerella cucumerina (Pc; Mock, Pc-I, Pc-II, Pc-III). F1 and F2 plants were analysed for resistance against the same pathogen. Lesion diameters were determined in 4 leaves/plant at 15 days post inoculation (dpi) and the average lesion diameter per plant was used as statistical unit of replication. Boxplots show the interquartile range (IQR; box) $\pm 1.5 x \mathrm{IQR}$ (whiskers), including median (horizonal line) and replication units (dots). (a) TAR against $P c$ in F1 progeny. Data represent lesion diameters (mm) of plants within $\mathrm{F} 1$ populations from similarly treated parents $(\mathrm{n}=40)$. Different letters indicate statistically significant differences (Welch ANOVA + Games-Howell test, $\alpha=0.05$ ). Data for individual F1 populations are shown in Fig. S3a. (b) TAR against $P c$ in F2 progeny after a stress-free F1 generation. Data represent lesion diameters of plants within F2 populations that share a common parental ancestor $(\mathrm{n}=20)$. Different letters indicate statistically significant differences (ANOVA + Tukey post-hoc test; $\alpha=0.05$ ). Data for individual F2 populations are shown in Fig. S3b.

Fig. 5. Lack of salt-elicited TAR in matched environments. Parental plants had been exposed to different stress intensities by soil salinity ( $\mathrm{NaCl}$; Mock, S-I, S-II, S-III). Salt tolerance of F1 and F2 plants was quantified by root growth reduction (\%) over a 5-day period on $\mathrm{NaCl}$-containing agar medium relative to the average root growth on agar medium without $\mathrm{NaCl}$. Boxplots show the interquartile range (IQR; box) \pm 1.5xIQR (whiskers), including median (horizonal line) and replication units (dots). (a) Unaltered tolerance of $\mathrm{F} 1$ plants to 50 and $100 \mathrm{mM} \mathrm{NaCl}$. Data represent growth reduction percentages of single plants within F1 populations from similarly treated parents $(n=60)$. ns: no statistically significant differences (ANOVA; $\alpha=0.05$ ). Root growth data for individual F1 populations are shown in Fig. S4a; root tolerance data for individual F1 populations are shown in Fig. S4c. (b) Unaltered tolerance of F2 plants to 50 and $100 \mathrm{mM}$ $\mathrm{NaCl}$ after one stress-free $\mathrm{F} 1$ generation. Data represent growth reduction percentages of single plants within F2 populations that share a common parental ancestor $(n=18-20)$. ns: no statistically significant differences (ANOVA; $\alpha=0.05$ ). Root growth data for individual F2 populations are shown in Fig. S4b; root tolerance data for individual F2 populations are shown in Fig. S4d.

Fig. 6. Costs and benefits of TAR in mismatched environments. Parental plants had been exposed to different stress severities by Pst (green), Pc (blue) or soil salinity (orange). F1 plants were tested for resistance against different stresses than the parental stress. (a) Increased $P c$ susceptibility in F1 progeny from Pst-exposed parents. Box plots show lesion diameters $(\mathrm{mm})$ of plants within F1 populations from similarly treated parental plants $(\mathrm{n}=76-80)$. See legend of Fig. 4 for details. Different letters indicate 
statistically significant differences between parental treatments (ANOVA + Tukey post-hoc test; $\alpha=0.05$ ). Data for individual F1 populations are shown in Fig. S5a. (b) Reduced salt tolerance in F1 progeny from Pst-exposed parents. Box plots show root growth reduction percentages by $50 \mathrm{mM} \mathrm{NaCl}$ of plants within F1 populations from similarly treated parental plants $(\mathrm{n}=40)$. See legend of Fig. $\mathbf{5}$ for details. Different letters indicate statistically significant differences (ANOVA + Tukey post-hoc test; $\alpha=0.05$ ). Root growth data for individual $\mathrm{F} 1$ populations at 0,50 and $100 \mathrm{mM} \mathrm{NaCl}$ are shown in Fig. S5b; tolerance data for individual $\mathrm{F} 1$ populations to 50 and $100 \mathrm{mM} \mathrm{NaCl}$ are shown in Fig. S5c. (c) Increased Hpa susceptibility in F1 progeny from $P c$-exposed parents. Stacked bars show leaf frequency distributions across Hpa resistance classes within F1 populations from similarly treated parents $(\mathrm{n}=400-500)$. See legend of Fig. 3b for details. Different letters indicate statistically significant differences (pairwise Fisher's exact tests + Bonferroni FDR, $\alpha=0.05$ ). Data for individual F1 populations are shown in Fig. S6a. (d) Unaltered salt tolerance in $\mathrm{F} 1$ progeny from $P c$-exposed parents. Box plots show root growth reduction percentages by $50 \mathrm{mM} \mathrm{NaCl}$ of plants within $\mathrm{F} 1$ populations from similarly treated parental plants $(\mathrm{n}=47)$. See legend of Fig. 5 for details. ns: no statistically significant differences (ANOVA; $\alpha=0.05$ ). Root growth data for individual $\mathrm{F} 1$ populations at 0,50 and $100 \mathrm{mM} \mathrm{NaCl}$ are shown in Fig. S6B; tolerance data for individual F1 populations to 50 and $100 \mathrm{mM} \mathrm{NaCl}$ are shown in Fig. S6c. (e) Non-specific TAR against Hpa in F1 progeny from $\mathrm{NaCl}$-exposed parents. Stacked bars show leaf frequency distributions across Hpa resistance classes within $\mathrm{F} 1$ populations from similarly treated parents $(\mathrm{n}=350-800)$. See legend of Fig. $\mathbf{3 b}$ for details. Different letters indicate statistically significant differences (Pairwise Fisher's exact tests + Bonferroni FDR, $\alpha=0.05$ ). Data for individual F1 populations are shown in Fig. S7a. (f) Non-specific TAR against Pc in F1 progeny from $\mathrm{NaCl}$-treated parents. Box plots show lesion diameters $(\mathrm{mm})$ of plants within $\mathrm{F} 1$ populations from similarly treated parental plants $(\mathrm{n}=30-60)$. See legend of Fig. 4 for details. Different letters indicate statistically significant differences between parental treatments (ANOVA + Tukey post-hoc test; $\alpha=0.05$ ). Data for individual F1 populations are shown in Fig. S7b.

\section{Supplementary Figures and Table}


(a)

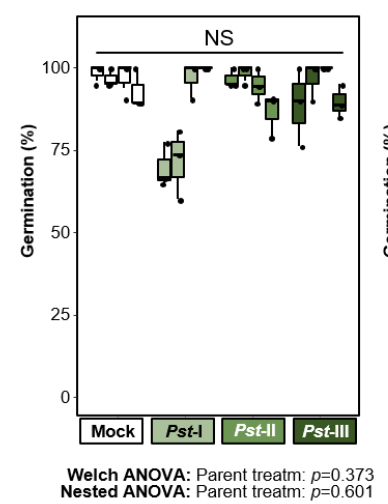

(b)

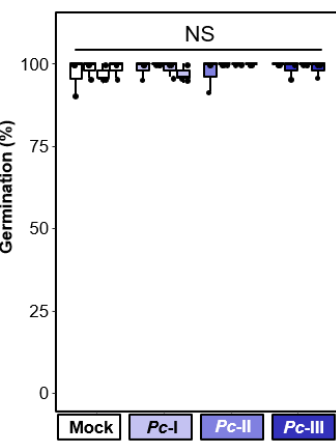

Welch ANOVA: Parent treetm: $0=0.308$ Nested ANOVA: Parent treatm: $p=0.340$ (c) Parents S - F1

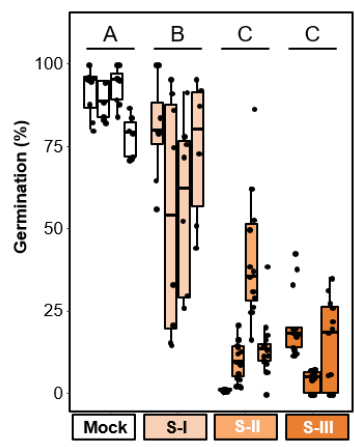

Welch ANOVA: Parent treatm: $p<0,0001$ Nested ANOVA: Parent treatm: $p<0.0001$

(d)

Parents S - F2

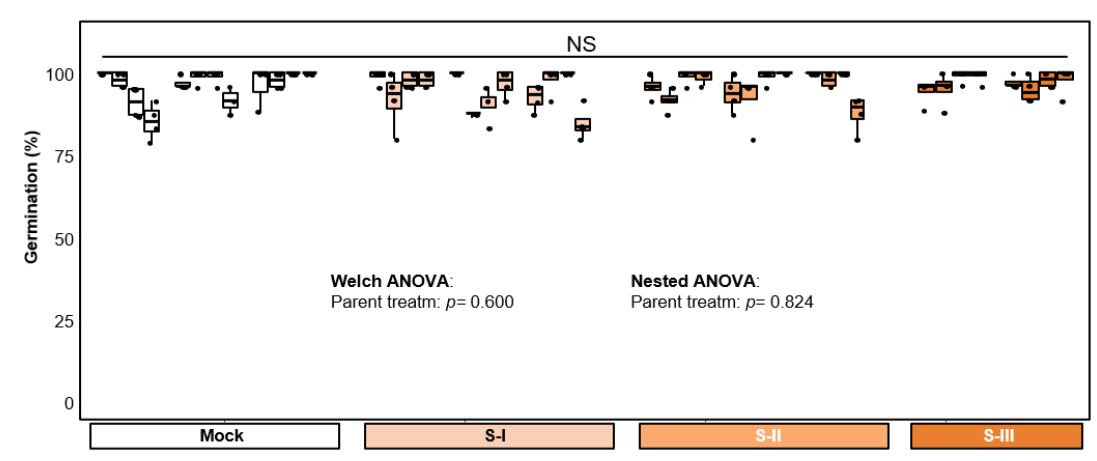

Figure 7: This is a caption

Fig. S1: Impacts of (a)biotic stresses on seed viability from individual plants. See legend of Fig. 2c for details. Boxplots show the interquartile range (IQR; box) $\pm 1.5 x I Q R$ (whiskers), including median (horizonal line) and replication units (dots). Data represent germination percentages of $\mathrm{F} 1$ seed batches from Pst-exposed parents (green, (a); n=3-4), F1 seeds from Pc-exposed parents (blue, (b); n=3-4), F1 seeds from salt-exposed parents (orange, $(\mathbf{c}) ; \mathrm{n}=8-22)$ and $\mathrm{F} 2$ seeds from salt-exposed parents after one stress-free F1 generation (orange, $(\mathrm{d}) ; \mathrm{n}=4-5)$. $P$-values indicate statistical significance of parent treatment by Welch ANOVA of pooled populations from similarly treated parent plants (F1) or a common parental ancestor (F2), as well as nested ANOVA with individual F1/F2 population as random factor. Different letters indicate statistically significant differences between pooled populations (Welch ANOVA + Games-Howell post-hoc test; $\alpha=0.05$; NS: no statistically significant differences). 
(a)

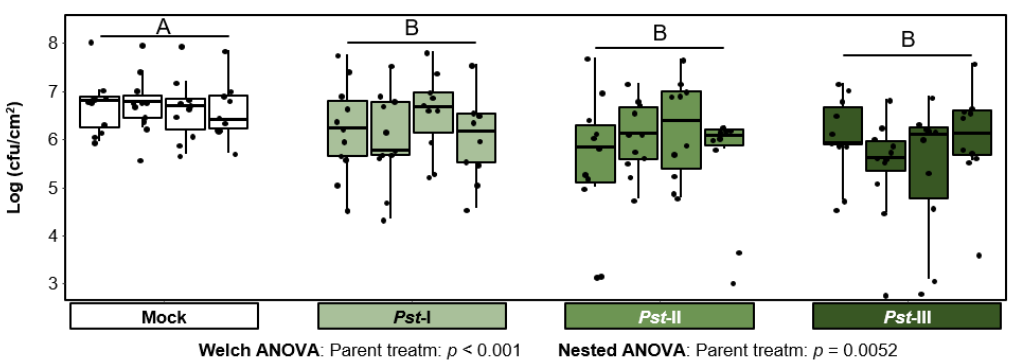

(d)
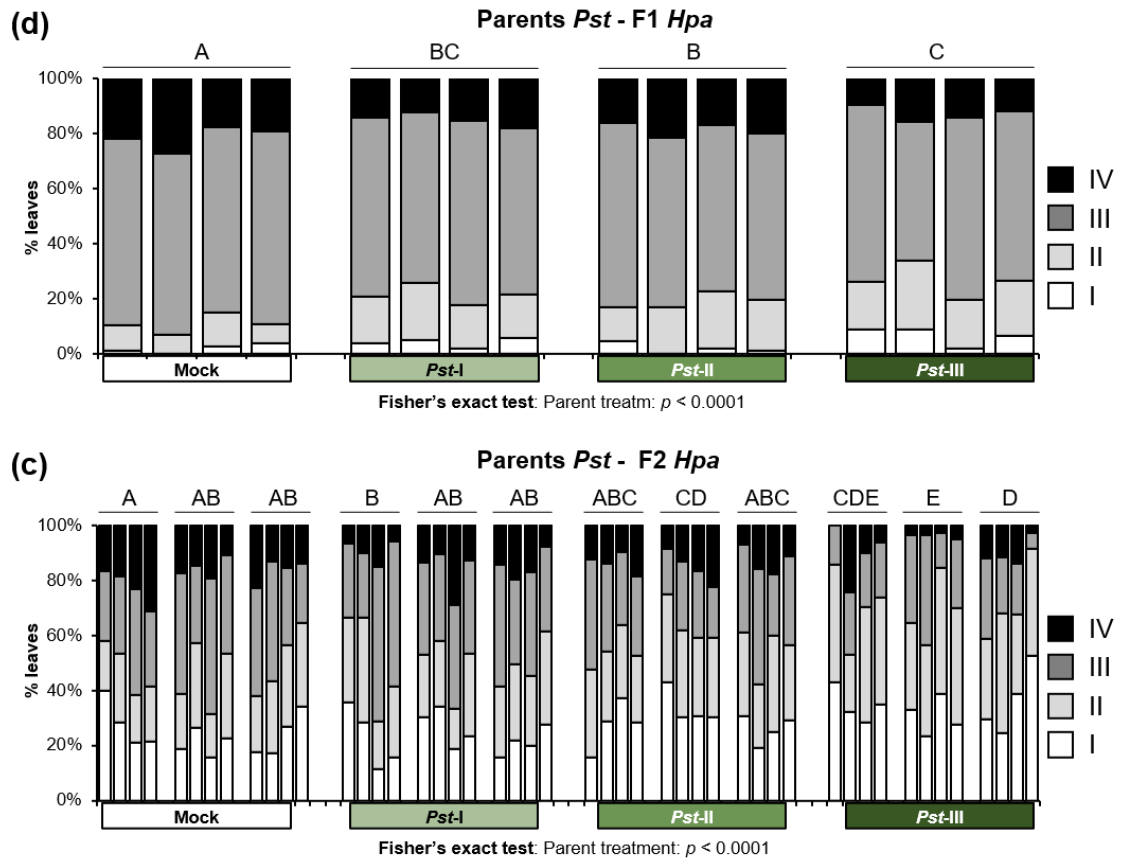

Figure 8: This is a caption

Fig. S2. TAR in individual F1 and F2 populations from Pst-treated parents in matched environments. (a) Pst-elicited TAR against Pst in F1 plants at 3 dpi. See legend of Fig. 3a for details. Boxplots show the interquartile range (IQR; box) $\pm 1.5 x \mathrm{IQR}$ (whiskers), including median (horizonal line) and replication units (dots). Data represent ${ }^{10} \mathrm{Log}$-transformed bacterial titres (Log $\left.\mathrm{cfu} \mathrm{cm}^{-2} ; \mathrm{n}=10-12\right)$ in leaves of single plants within individual F1 populations. $P$-values indicate statistical significance of parent treatment by Welch ANOVA of pooled F1 populations from similarly treated parents, and nested ANOVA with individual F1 population as random factor, respectively. Different letters indicate statistically significant differences between pooled F1 populations from similarly treated parents (Welch ANOVA + Games-Howell post-hoc test; $\alpha=0.05)$. (b) Pst-elicited TAR against $H p a$ in F1 plants at 6 dpi. See legend of Fig. 3b for details. Stacked bars show leaf frequency distributions across Hpa resistance classes within individual F1 populations $(n=70-250)$. $P$-value indicates statistical significance of the parent treatment (Fisher's exact test). Different letters indicate statistically significant differences between pooled F1 populations from similarly treated parents (pairwise Fisher's exact tests + Bonferroni FDR, $\alpha=0.05$ ). (c) Pst-elicited TAR against $H p a$ in F2 plants at 6 dpi after one stress-free F1 generation. See legend of Fig. 3c for details. Stacked bars show leaf frequency distributions across $H p a$ resistance classes within individual F2 populations $(\mathrm{n}=55-100) . \quad P$-value indicates statistical significance of parent treatment (Fisher's exact tests). Different 
letters indicate statistically significant differences between pooled F2 populations from a common parental ancestor (pairwise Fisher's exact tests + Bonferroni FDR; $\alpha=0.05$ ).
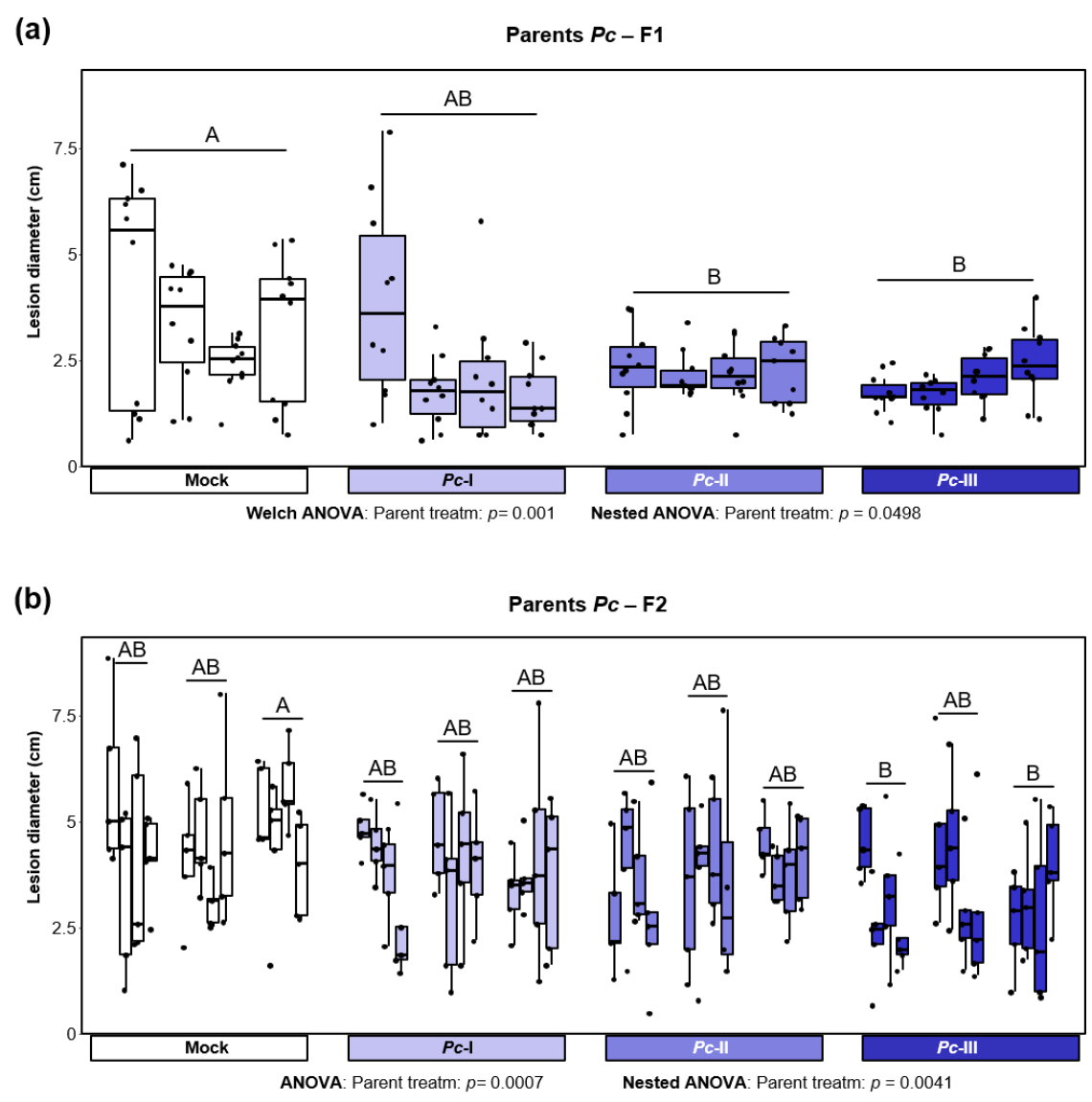

Figure 9: This is a caption

Fig. S3. TAR in individual F1 and F2 populations from $P c$-exposed parents in matched environments. Data represent lesion diameters $(\mathrm{mm})$ of plants within individual populations at 15 dpi. See legend of Fig. 4 for details. Boxplots show the interquartile range (IQR; box) $\pm 1.5 x I Q R$ (whiskers), including median (horizonal line) and replication units (dots). (a) $P c$-elicited TAR against $P c$ in F1 plants (n=10). $P$-values indicate statistical significance of parent treatment by Welch ANOVA of pooled F1 populations from similarly treated parents, and nested ANOVA with F1 population as random factor, respectively. Different letters indicate statistically significant differences between pooled F1 populations from similarly treated parents (Welch ANOVA + Games-Howell post-hoc test; $\alpha=0.05$ ). (b) $P c$-elicited TAR against $P c$ in F2 plants $(\mathrm{n}=5) . P$-values indicate statistical significance of parent treatment by ANOVA of pooled F2 populations from a common parental ancestor, and nested ANOVA with F2 population as random factor, respectively. Different letters indicate statistically significant differences between pooled F2 populations from a common parental ancestor (ANOVA + Tukey post-hoc test; $\alpha=0.05$ ). 

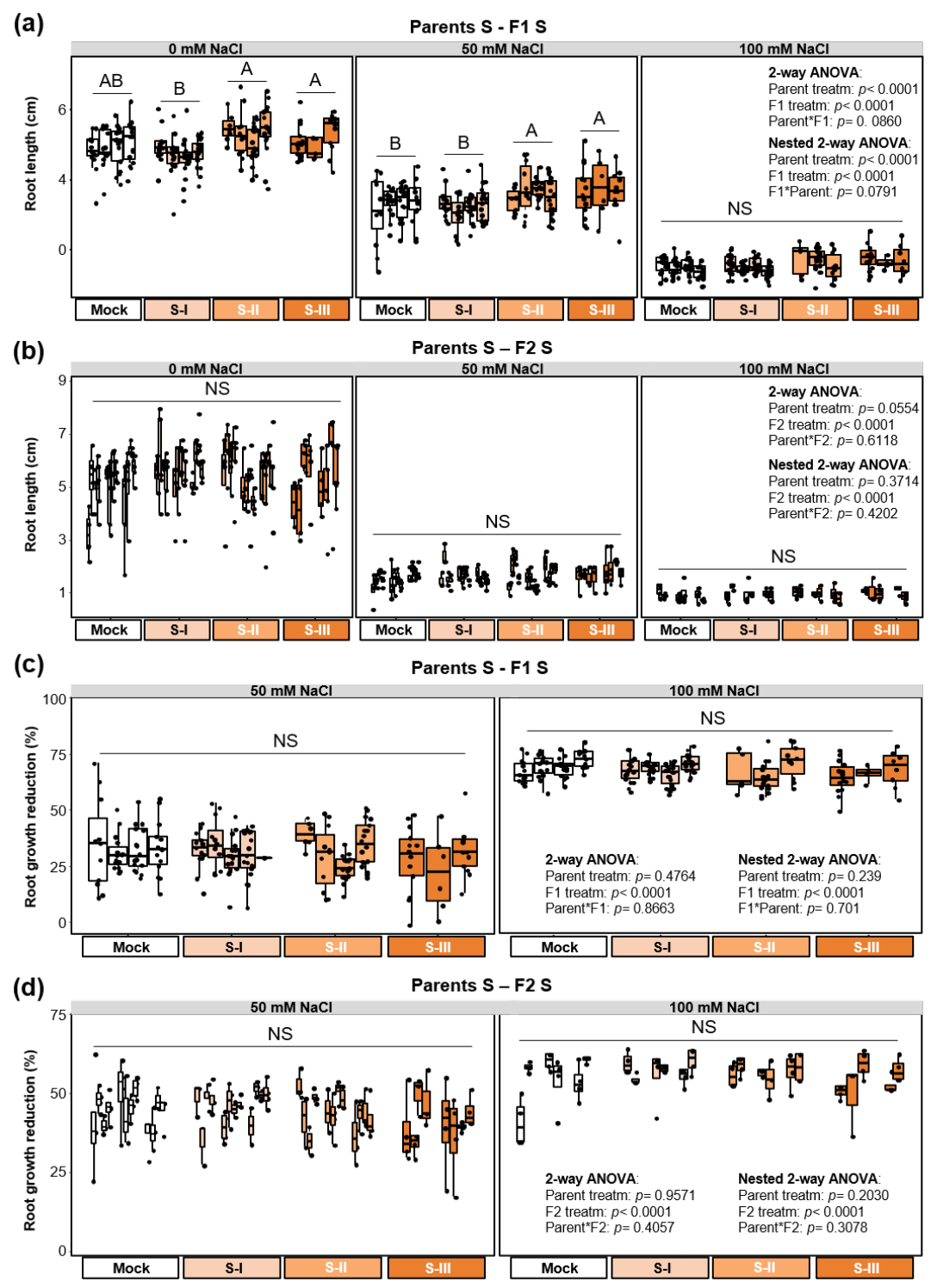

Figure 10: This is a caption

Fig4. Transgenerational effects of soil salinity on root growth and salt tolerance in individual F1 and F2 populations and matched environments. All boxplots show the interquartile range (IQR; box) $\pm 1.5 x \mathrm{IQR}$ (whiskers), including median (horizonal line) and replication units (dots). (a) Root growth of F1 plants at 0,50 and $100 \mathrm{mM} \mathrm{NaCl}$. Data represent root growth values $(\mathrm{cm})$ of plants within individual F1 populations over a 5 -day period $(n=15) . P$-values indicate statistical significance of parent treatment, F1 treatment and interaction by 2-way ANOVA of pooled F1 populations from similarly treated parents, and nested 2-way ANOVA with F1 population as random factor, respectively. For each $\mathrm{NaCl}$ concentration, different letters indicate statistically significant differences between pooled F1 populations from similarly treated parents (ANOVA + Tukey post-hoc test; $\alpha=0.05$; NS: no significant differences). (b) Root growth of $\mathrm{F} 2$ plants at 0,50 and $100 \mathrm{mM} \mathrm{NaCl}$ after one stress-free $\mathrm{F} 1$ generation. Data represent root growth values $(\mathrm{cm})$ of plants within individual F2 populations over a 5 -day period $(\mathrm{n}=4-5) . \quad P$-values indicate 
statistical significance of parent treatment, F2 treatment and interaction by 2-way ANOVA of pooled F2 populations from a common parental ancestor, and nested 2-way ANOVA with F2 population as random factor, respectively. NS: no statistically significant differences between pooled F2 populations from a common parental ancestor (ANOVA + Tukey post-hoc test; $\alpha=0.05$ ). (c) Tolerance of F1 plants to 50 and $100 \mathrm{mM}$ $\mathrm{NaCl}$. Tolerance was quantified by root growth reduction (\%) relative to the mean root growth at 0 $\mathrm{mM} \mathrm{NaCl}$ of the corresponding $\mathrm{F} 1$ population (Fig. S4a). Data represent growth reduction percentages of single plants within individual $\mathrm{F} 1$ populations $(\mathrm{n}=15) . \quad P$-values indicate statistical significance of parent treatment, F1 treatment and interaction by 2-way ANOVA of pooled F1 populations from similarly treated parents, and nested 2-way ANOVA with F1 population as random factor, respectively. NS: no statistically significant differences between pooled F1 populations from similarly treated parents (ANOVA + Tukey posthoc test; $\alpha=0.05$ ). (d) Tolerance of $\mathrm{F} 2$ plants to 50 and $100 \mathrm{mM} \mathrm{NaCl}$ after one stress-free $\mathrm{F} 1$ generation. Tolerance was quantified by root growth reduction relative to the mean root growth at $0 \mathrm{mM} \mathrm{NaCl}$ of the corresponding F2 population (Fig. S4b). Data represent growth reduction percentages of single plants within individual F2 populations $(\mathrm{n}=4-5)$. $P$-values indicate statistical significance of parent treatment, $\mathrm{F} 2$ treatment and interaction by 2 -way ANOVA of pooled F2 populations from a common parental ancestor, and nested 2-way ANOVA with F2 population as random factor, respectively. NS: no statistically significant differences between pooled F2 populations from a common parental ancestor (ANOVA + Tukey post-hoc test; $\alpha=0.05)$.
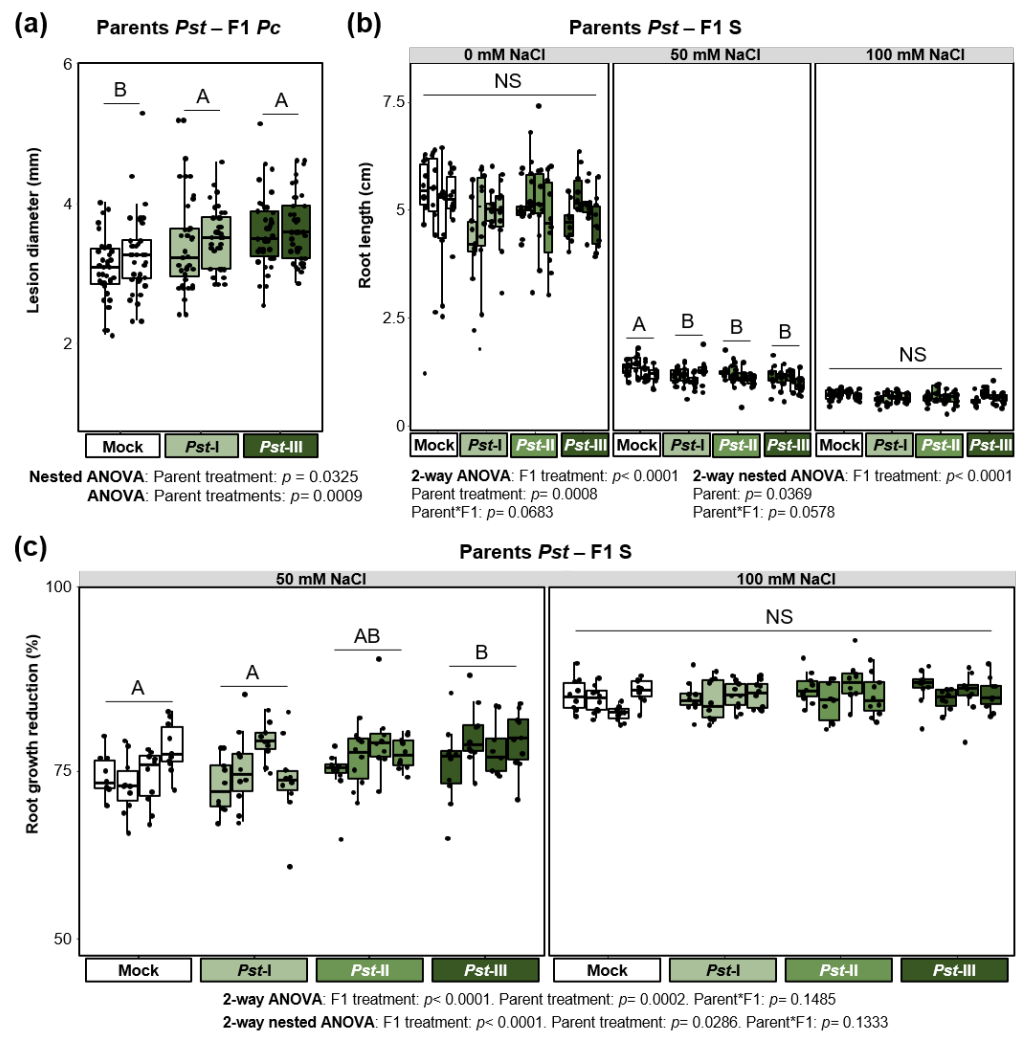

Figure 11: This is a caption 
Fig. S5. Costs of Pst-elicited TAR in individual F1 populations and mismatched environments. All boxplots show the interquartile range (IQR; box) $\pm 1.5 x I Q R$ (whiskers), including median (horizonal line) and replication units (dots). See legends to Fig. 6a,b for details. (a) Pc resistance of F1 plants from mock- and Pst-treated parents 6 dpi. Data represent lesion diameters $(\mathrm{mm})$ of plants within individual F1 populations $(\mathrm{n}=38-40) . P$-values indicate statistical significance of parent treatment by ANOVA of pooled F1 populations from similarly treated parents, and nested ANOVA with individual F1 population as random factor, respectively. Different letters indicate statistically significant differences between pooled F1 populations from similarly treated parents (ANOVA + Tukey post-hoc test; $\alpha=0.05$ ). (b) Root growth of F1 plants from mock- and Pst-treated parents at 0,50 and $100 \mathrm{mM} \mathrm{NaCl}$. Data represent root growth values $(\mathrm{cm})$ of plants within individual F1 populations over a 5 -day period $(\mathrm{n}=10) . P$-values indicate statistical significance of parent treatment, F1 treatment and interaction by 2-way ANOVA of pooled F1 populations from similarly treated parental plants, and nested 2-way ANOVA with F1 population as random factor, respectively. For each $\mathrm{NaCl}$ concentration, different letters indicate statistically significant differences between pooled F1 populations from similarly treated parents (ANOVA + Tukey post-hoc test; $\alpha=0.05$; NS: no statistically significant differences). (c) Tolerance of F1 plants from mock- and Pst-treated parents to 50 and $100 \mathrm{mM}$ $\mathrm{NaCl}$. Tolerance was quantified by root growth reduction (\%) relative to the mean root growth value at 0 $\mathrm{mM} \mathrm{NaCl}$ of the corresponding $\mathrm{F} 1$ population (Fig. S5b). Data represent root growth reduction percentages of single plants within individual $\mathrm{F} 1$ populations $(\mathrm{n}=10) . P$-values indicate statistical significance of parent treatment, F1 treatment and interaction by 2-way ANOVA of pooled F1 populations from similarly treated parents, and by nested 2-way ANOVA with F1 population as random factor, respectively. For each $\mathrm{NaCl}$ concentration, different letters indicate statistically significant differences between pooled $\mathrm{F} 1$ populations from similarly treated parents (ANOVA + Tukey post-hoc test; $\alpha=0.05$; NS: no significant differences).
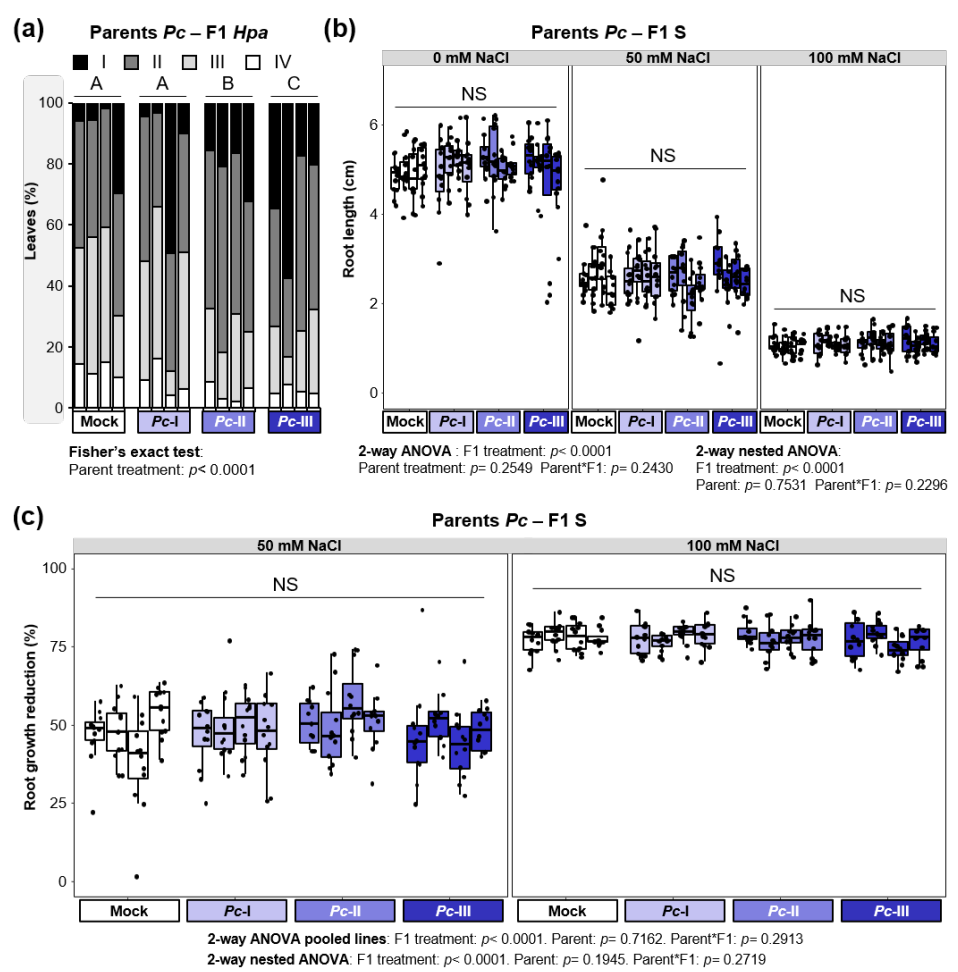

Figure 12: This is a caption

Fig. S6. Costs of Pc-elicited TAR in individual F1 populations and mismatched environments. 
See legends of Fig. 6c,d for details. (a) Hpa resistance in F1 plants from $P c$-exposed parents. Stacked bars show leaf frequency distributions across $H p a$ resistance classes within F1 populations from similarly treated parents $(\mathrm{n}=80-130)$. $P$-value indicates statistical significance of parental treatment (Fisher's exact test). Different letters indicate statistically significant differences between pooled F1 populations from similarly treated parents (Pairwise Fisher's exact tests + Bonferroni FDR; $\alpha=0.05$ ). (b) Root growth of F1 plants from mock- and $P c$-treated parents at 0,50 and $100 \mathrm{mM} \mathrm{NaCl}$. Boxplots show the interquartile range (IQR; box) $\pm 1.5 \mathrm{xIQR}$ (whiskers), including median (horizonal line) and replication units (dots). Data represent root growth values $(\mathrm{cm})$ of single plants within individual $\mathrm{F} 1$ populations over a 5 -day period $(\mathrm{n}=10)$. $P$-values indicate statistical significance of parent treatment, F1 treatment and interaction by 2-way ANOVA of pooled F1 populations from similarly treated parents, and nested 2-way ANOVA with F1 population as random factor, respectively. NS: no statistically significant differences between pooled F1 populations from similarly treated parents (ANOVA + Tukey post-hoc test; $\alpha=0.05$ ). (c) Tolerance of F1 plants from mock- and Pctreated parents to 50 and $100 \mathrm{mM} \mathrm{NaCl}$. Tolerance was quantified by root growth reduction (\%) relative to the mean root growth value at $0 \mathrm{mM} \mathrm{NaCl}$ of the corresponding $\mathrm{F} 1$ population (Fig. S6b). Boxplots show the interquartile range (IQR; box) $\pm 1.5 x \mathrm{IQR}$ (whiskers), including median (horizonal line) and replication units (dots). Data represent root growth reduction percentages of single plants within individual F1 populations. $P$-values indicate statistical significance of parent treatment, F1 treatment and interaction by 2 -way ANOVA of pooled F1 populations from similarly treated parents, and nested 2-way ANOVA with F1 population as random factor, respectively. NS: no statistically significant differences between pooled F1 populations from similarly treated parents (ANOVA + Tukey post-hoc test; $\alpha=0.05$ ).
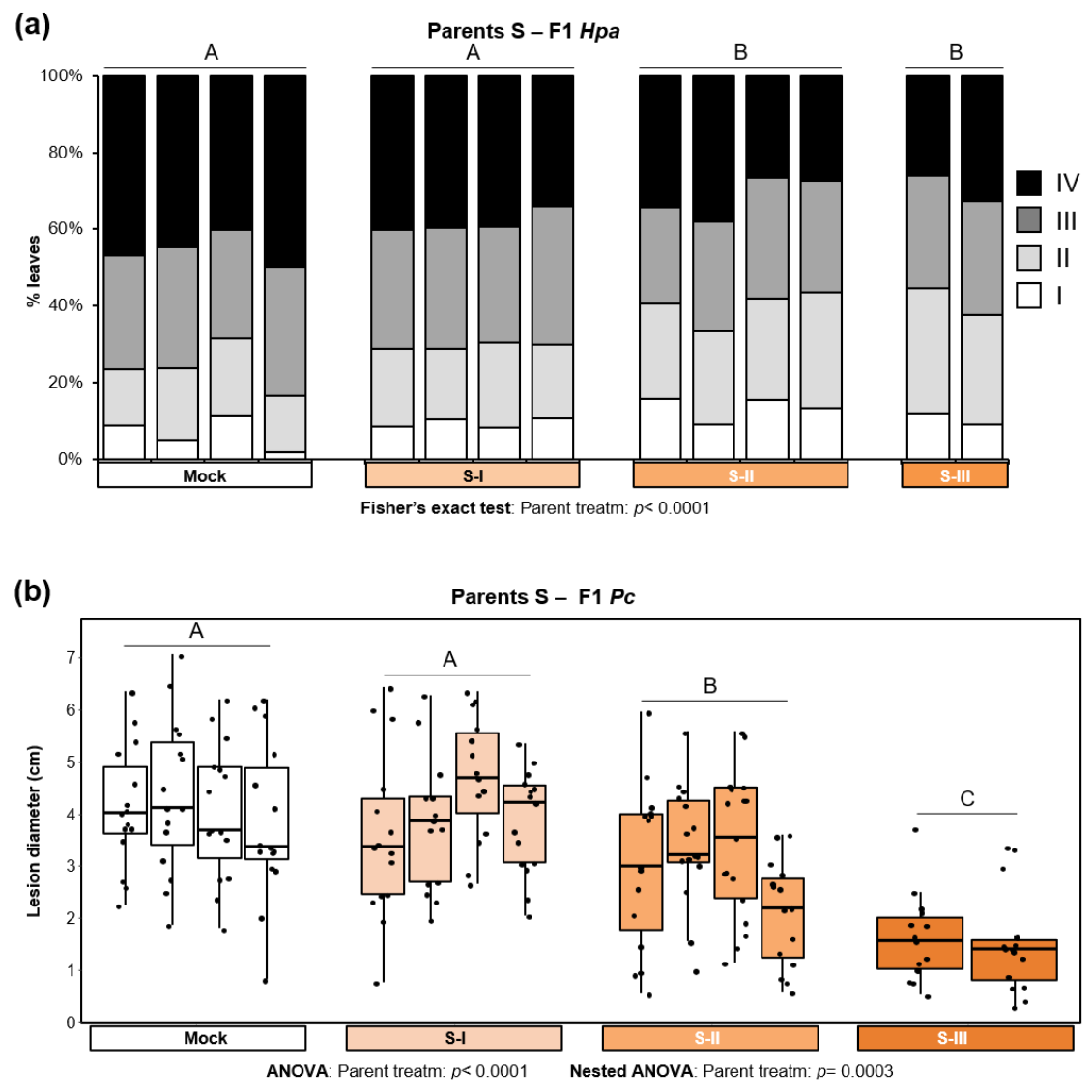

Figure 13: This is a caption 
Fig. S7. Non-specific TAR by soil salinity against $P s t$ and $P c$ in individual F1 populations and mismatched environments. See legend of Fig. 6e,f for details. (a) Non specific TAR against Hpa in F1 plants. Stacked bars show leaf frequency distributions across Hpa resistance classes within individual F1 populations (n=100-225). $P$-value indicates statistical significance of parental treatment (Fisher's exact test). Different letters indicate statistically significant differences between pooled F1 populations from similarly treated parental plants (pairwise Fisher's exact tests + Bonferroni FDR; $\alpha=0.05$ ). (b) Non-specific TAR against $P c$ in F1 plants. Boxplots show the interquartile range (IQR; box) $\pm 1.5 x \mathrm{IQR}$ (whiskers), including median (horizonal line) and replication units (dots). Data represent lesion diameters $(\mathrm{mm})$ of plants within individual $\mathrm{F} 1$ populations $(\mathrm{n}=15) . P$-values on the right indicate statistical significance of parent treatment by ANOVA of pooled F1 populations from similarly treated parental plants, and nested ANOVA with individual F1 population as random factor, respectively. Different letters indicate statistically significant differences between pooled F1 populations from similarly treated parents (ANOVA + Tukey post-hoc test; $\alpha=0.05)$.

Table S1. Collection of Arabidopsis F1 and F2 populations (Col-0) that in the parental generation had been subjected to varying stress intensities by $P s t, P c$ or soil salinity.

\begin{tabular}{|c|c|c|c|}
\hline Parental treatment & Generation & No. of lines & Code \\
\hline Pst-Mock & F1 & 4 lines & Pst-0, 1-4 \\
\hline Pst-Low & F1 & 4 lines & Pst-I, 1-4 \\
\hline Pst-Medium & $\mathrm{F} 1$ & 4 lines & Pst-II, 1-4 \\
\hline Pst-High & F1 & 4 lines & Pst-III, 1-4 \\
\hline Pst-Mock & $\mathrm{F} 2$ & 12 lines & Pst-0 M2, 1-12 \\
\hline Pst-Low & F2 & 12 lines & Pst-I M2, 1-12 \\
\hline Pst-Medium & F2 & 12 lines & Pst-II M2, 1-12 \\
\hline Pst-High & $\mathrm{F} 2$ & 12 lines & Pst-III M2, 1-12 \\
\hline F1 Pc-Mock & F1 & 4 lines & $P c-0,1-4$ \\
\hline F1 $P c$-Low & F1 & 4 lines & $P c-\mathrm{I}, 1-4$ \\
\hline F1 $P c$-Medium & F1 & 4 lines & $P c$-II, 1-4 \\
\hline F1 Pc-High & F1 & 4 lines & $P c$-III, 1-4 \\
\hline F2 Pc-Mock & F2 & 12 lines & $P c-0 \mathrm{M} 2,1-12$ \\
\hline F2 Pc-Low & F2 & 12 lines & $P c-\mathrm{I}$ M2, 1-12 \\
\hline F2 Pc-Medium & $\mathrm{F} 2$ & 12 lines & $P c-$ II M2, 1-12 \\
\hline F2 Pc-High & $\mathrm{F} 2$ & 12 lines & $P c$-III M2, 1-12 \\
\hline F1 Salt-Mock & $\mathrm{F} 1$ & 4 lines & S-0, 1-4 \\
\hline F1 Salt-Low & F1 & 4 lines & S-I, 1-4 \\
\hline F1 Salt-Medium ${ }^{1}$ & F1 & 4 lines & S-II, 1-4 \\
\hline F1 Salt-High ${ }^{1}$ & $\mathrm{~F} 1$ & 4 lines & S-III, 1-4 \\
\hline F2 Salt-Mock & $\mathrm{F} 2$ & 12 lines & $\mathrm{S}-0 \mathrm{M} 2,1-12$ \\
\hline F2 Salt-Low & F2 & 12 lines & S-I M2, 1-12 \\
\hline F2 Salt-Medium & F2 & 12 lines & S-II M2, 1-12 \\
\hline F2 S-High & F2 & 8 lines & S-III M2, 1-12 \\
\hline
\end{tabular}

\section{Supplementary Methods}




\section{General growth conditions.}

After stratification in water and darkness at $4^{\circ} \mathrm{C}$ for $2-3$ days, seeds were sown on Jiffy- 7 peat pellets. All plants were initially grown under short-day conditions ( 8.5 hour -h- light $/ 15.5 \mathrm{~h}$ darkness) at $21{ }^{\circ} \mathrm{C}, 60 \%$ relative humidity $(\mathrm{RH})$ and $100-125 \mu \mathrm{mol} \mathrm{s}{ }^{-1} \mathrm{~m}^{-2}$ light intensity) and watered to saturation by flooding the trays for $0.5 \mathrm{~h}$ and removing the excess of water afterwards (3 times/week). After the stress exposure, parental plants (7.5-weeks-old) were moved to long-day conditions (16 h light $/ 8 \mathrm{~h}$ darkness) to trigger flowering and set seed. For quantification of disease resistance in F1/F2 populations, plants were grown in individual pots in a randomised block design, and regularly rotated within and between blocks within the climate chamber (3 times/week) to prevent positional effects. For the quantification of $\mathrm{NaCl}$ tolerance, agar plates containing multiple F1/F2 populations were rotated with similar frequency.

\section{Microbes and inoculation protocols.}

Pseudomonas syringae pv. tomato strain DC3000 was cultivated from a frozen glycerol stock for $48 \mathrm{~h}$ on King's B (KB) agar plates, supplemented with $50 \mu \mathrm{g} / \mathrm{ml}$ rifampicin (Sigma-Aldrich, R3501). Cells were collected from agar plates, resuspended and washed in $10 \mathrm{mM} \mathrm{MgSO}_{4}$ before adjusting the optical density spectrophotometrically (OD600) For stress induction, cells were adjusted to to $5 \times 10^{7}$ colony-forming units $(\mathrm{CFU}) / \mathrm{mL}$ and supplemented with $0.015 \%$ Silwet L-77 (Lehle Seeds NC0138454) prior to spraying onto the rosettes. Mock inoculation was performed by spraying equal amounts of $10 \mathrm{mM} \mathrm{MgSO} 4+0.015 \%$ Silwet L-77). For quantification of $P s t$ resistance in F1/F2 progeny, the inoculum was adjusted to $2 \times 10^{5} \mathrm{CFU} / \mathrm{mL}$ and syringe-infiltrated into 4 leaves/plant of approximate similar age. Plants were kept at $100 \% \mathrm{RH}$ for 1-2 h immediately after inoculation. Plectosphaerella cucumerina strain BMM $(P c)$ was cultivated on halfstrength Potato Dextrose Agar (BD Difco, BD-213400) for 3.5 weeks in the dark. Spores were resuspended from agar plates in water and filtered through 2 layers of Miracloth (Merck, 475855-1R) to remove mycelium debris. $P c$ inoculum was adjusted to $10^{6}$ spores $/ \mathrm{ml}$ in water, using a Neubauer haemocytometer. To ensure necrotrophic infection by the fungus, inoculation was performed by placing $6 \mu \mathrm{l}$ droplets $\left(10^{6} \mathrm{spores} / \mathrm{ml}\right)$ onto fully expanded leaves of approximate similar age; mock inoculum was performed by of applying $6 \mu$ l water droplets. The obligate biotrophic Oomycete Hyaloperonosopora arabidopsidis strain WACO9 was maintained and bulked on hypersusceptible NahG plants (Ws-0, Syngenta Agribusiness Biotechnology Research, Line $3 \mathrm{~A}$ ). Sporulating plants were collected in $15-\mathrm{mL}$ falcon tubes containing demineralised water and gently shaken to extract conidiospores. The suspension was then filtered through 2 layers of Miracloth and adjusted to $10^{5}$ conidiospores/mL, using a Neubauer haemocytometer. Three-week-old plants were inoculated by spraying the spore suspension onto the shoots, after which plants were maintained at $100 \% \mathrm{RH}$.

\section{Analysis of relative growth rate.}

Relative growth rate (RGR) analysis was based quantification of green leaf area (GLA) before and after stress treatments. Digital photos (Canon, 500D 15MP) were taken before and after the stress treatment. Digital image analysis of GLA was performed using Adobe Photoshop 6.0. Green pixels corresponding to GLA were selected using a combination of "magic wand" and "lasso" tools and converted into $\mathrm{mm}^{2}$. For each plant, the following formula was used to calculate RGR, where GLA 2 and GLA 1 are GLA values before and after stress exposure, respectively, and $(t 2-t 1)$ represents the time-window (d):

RGR values were determined for 5- 6 plants per treatment and normalized to the average RGR value of non-stressed plants (mock treatment; 100\%). Reproductive fitness was estimated by seed production and seed viability as described in the Supplementary Methods.

\section{Seed production and seed viability assays.}


To estimate reproductive fitness, seeds from 5- 6 plants per stress treatment were collected in Aracons (Lehle seeds) and weighed. Seed weights for each plant were converted to numbers of seeds, based on the mass of 100 counted seeds $(\sim 1.2 \mathrm{mg})$. Seed viability was determined after sterilisation on agar plates (see for details). Seed viability was quantified on 0.2x Murashige and Skoog (MS) agar (Duchefa, M0221), containing $1 \%$ sucrose and $6 \mathrm{~g} / \mathrm{L}$ agar $(\mathrm{pH}=5.7$, adjusted with $\mathrm{KOH})$. Vapour-phase sterilization was performed by incubating seeds for $4 \mathrm{~h}$ in open Eppendorf tubes inside a glass vacuum desiccator $(10.5 \mathrm{~L})$, in which $100 \mathrm{ml}$ of bleach (Jantex, R-GG183) and $3 \mathrm{ml}$ of $\mathrm{HCl}$ were mixed to produce chlorine gas. Plates were stratified at $4^{\circ} \mathrm{C}$ in the dark for $3 \mathrm{~d}$ and transferred to short-day growth conditions. Seed germination rates were determined in 3-17 replicate plates/population ( 25 seeds/plate) at $5 \mathrm{~d}$ after stratification. Seeds were considered germinated when green cotyledons were visible.

\section{Pst resistance assays.}

Bacterial growth in syringe-inoculated leaves (see above) was quantified at 3 days post inoculation (dpi) by collecting 4 leaf discs/plant in 1.5-mL tubes with $600 \mu \mathrm{L} 10 \mathrm{mM} \mathrm{MgSO}_{4}$, using a cork borer $(0.75 \mathrm{~cm}$ diameter). Leaf discs were homogenised in the tubes using plastic pestles and transferred to 96-wells microtiter plates (Costar $\AA$ ) for serial dilutions in $10 \mathrm{mM} \mathrm{MgSO}_{4}$. Twelve samples in each plate were serial-diluted 8 times (5-fold dilutions) and plated onto selective KB agar plates, containing 50mg/mL Rifampicin (SigmaAldrich, R3501), using 96-wells Scienceware@ replicator (Sigma-Aldrich). For each 96-wells plate, 2 technical replicates were plated onto separate $\mathrm{KB}$ agar plates and incubated at $28^{\circ} \mathrm{C}$ for 2 days before counting CFUs. For each biologically replicated sample $(\mathrm{n}=10-12)$, bacterial CFUs were averaged between two technical replicates and 2-3 serial dilutions. For each plant, bacterial CFUs were normalised to its leaf area $\left(\mathrm{mm}^{2}\right)$.

\section{Hpa resistance assays.}

Inoculated shoots were collected at 6 dpi in trypan blue solution $(0.067 \% \mathrm{w} / \mathrm{v}$ trypan blue, $33 \% \mathrm{w} / \mathrm{v}$ phenol, $33 \%$ v.v glycerol, $33 \%$ v.v DL-lactic acid, supplemented with 2 volumes $100 \%$ ethanol), boiled for 60 sec, kept at room temperature (RT) for $15 \mathrm{~min}$, boiled again for $30 \mathrm{sec}$, and incubated at RT for $3 \mathrm{~h}$. Shoots were de-stained in $60 \%$ Chloral hydrate (Sigma-Aldrich, 23100) for at least $12 \mathrm{~h}$ before scoring. Stained leaves were scored under a stereomicroscope by assigning each leaf to one of the 4 different colonisation classes, which are based on distinct stages of $H p a$ pathogenesis: class I: no hyphal colonisation visible; class II: hyphal colonization without conidiophores; class III: hyphal colonization with conidiophore formation; class IV: extensive hyphal colonization with conidiophores and sexual oospores. Resistance scoring was based on $\sim 60$ seedlings/population ( $\sim 240$ leaves/ population) for F1 plants and $\sim 20$ seedlings/population ( $\sim 80$ leaves/population) for F2 plants, representing $\sim 900$ leaves/parental stress treatment.

\section{$P c$ resistance assays.}

After droplet-inoculation of 4 leaves/plant of approximate similar age (see above), plants were kept at $100 \% \mathrm{RH}$ and monitored daily for disease progression, which appeared as necrotic lesions surrounded by wider chlorotic halos at the sites of inoculation. Fungal colonisation was quantified by average diameter of the tightly defined necrotic lesion area. The time-point of scoring varied between experiments and was determined when average lesion diameters in a subsample of 5-10 individuals from the control group (progeny of mock-inoculated plants) was $>3 \mathrm{~mm}$. Four lesions per plant were averaged and used as unit of statistical replication.

\section{Salt tolerance assays.}

Salt tolerance assays were performed as described previously (Verslues et al., 2006; Claeys et al., 2014) with 
modifications. Seeds were vapour-sterilized, stratified and germinated as described for the seed viability assays. At $5 \mathrm{~d}$ after moving the $0.2 \mathrm{x}$ MS plates to short-day light conditions, seedlings were transplanted onto new 0.2 x MS plates containing $0 \mathrm{mM}, 50 \mathrm{mM}$ or $100 \mathrm{mM} \mathrm{NaCl}$. To avoid contact of leaves with the (NaCl-containing) MS agar, seedlings were positioned along a straight line above which the agar was excised. The root tip was marked on the plate as a reference point to determine root growth. At $5 \mathrm{~d}$ after transplantation, the length of the newly formed root from the reference point was determined for each individual plant. Salt stress was quantified as the percentage root growth reduction in each individual plant relative to the average root length on $0 \mathrm{mM} \mathrm{NaCl}$ agar of the corresponding line.

\section{Statistical analysis.}

Continuous variables were analysed by general linear models. Residuals were first analysed for normal distributions, using Shakiro-Wilk tests and Q-Q plots. If residuals failed to show normal distributions, data were either arcsine-transformed (percentage data), or Box-Cox transformed, using the 'MASS' R package (MASS_7.3-51.5.tar.gz). Models were analysed for heteroscedasticity, using Levene's tests ('car' R package car_3.0-0.tar.gz-). When variances were confirmed to be homogeneous, effects of parent treatment were tested by ANOVA models ('nlme' R package - nlme_3.1-137.tar.gz). Statistical significance of parental treatment on F1 resistance against $P$. syringae (colony forming units; CFU) and P. cucumerina (lesion diameters; $\mathrm{mm}$ ) were determined by two separate models. In addition to ANOVA of pooled F1 lines from similarly treated parent plants, using 'aov' function in R base, we performed nested ANOVA with F1 line as random variable, using the 'Ime' (method = "REML") and 'anova.lme' function (type = "sequential"; adjustSigma $=$ FALSE) from the 'nlme' R package. Similarly, parental effects on F2 resistance were determined by ANOVA of pooled F2 populations from the same parent plant, as well as nested ANOVA with F2 population as random variable. When both models indicated a statistically significant effect of parent treatment, Tukey HSD post-hoc tests were performed to identify statistically significant differences between pooled F1 populations from similarly treated parent plants or between pooled F2 populations from the same parental ancestor, using the 'TukeyHSD' function in R base. If data continued to show heteroscedasticity after transformation, parental effects were studied by Welch ANOVA, followed by Games-Howell post-hoc tests, using the 'userfriendlyscience' $\mathrm{R}$ package (userfriendlyscience_0.7.2.tar.gz). Statistical effects of parental salt treatment on root length $(\mathrm{mm})$ and root length reduction (\% relative to $0 \mathrm{mM} \mathrm{NaCl}$ treatment) were analysed by two-way ANOVA, in order to separate the effects of parental salt treatment (induction) from progeny salt treatment (challenge). In all cases, type II models were used after having confirmed lack of statistically significant interactions between parent and progeny treatments. The statistical significance of parental effects on root length and root length reduction in F1 plants was determined by 2-way ANOVA of pooled F1 populations from similarly treated parent plants ('aov' function followed by 'Anova' function; type = "II"), as well as 2-way mixed-effect ANOVA with F1 population as random variable ('Imer' function of 'lme4' R package followed by 'Anova' function; type = "II"). Similarly, parental effects on root length and root length reduction in F2 plants were determined by 2-way ANOVA of pooled F2 populations from the same parent plant, as well 2-way mixed-effect ANOVA with F2 population as random variable. When both 2-way ANOVA models indicated a statistically significant effect by parental treatment, pooled F1 populations from similarly treated parent plants were analysed for statistically significant differences at each progeny salt concentration $(0 \mathrm{mM}$, $50 \mathrm{mM}$ and $100 \mathrm{mM}$ ), using ANOVA followed by Tukey HSD post-hoc tests. Categorical variables (leaf frequencies within Hpa colonisation classes) were analysed for statistical differences by Fisher's exact tests. Statistically significant effects by parental treatment were assessed by pooling populations derived from similarly treated parents (F1) or a common parental ancestor (F2) in the cross table. Statistical differences between population groups were determined by pairwise Fisher's exact tests after Bonferroni multiple testing correction, using the R package 'fifer' (fifer_1.1.tar.gz). 